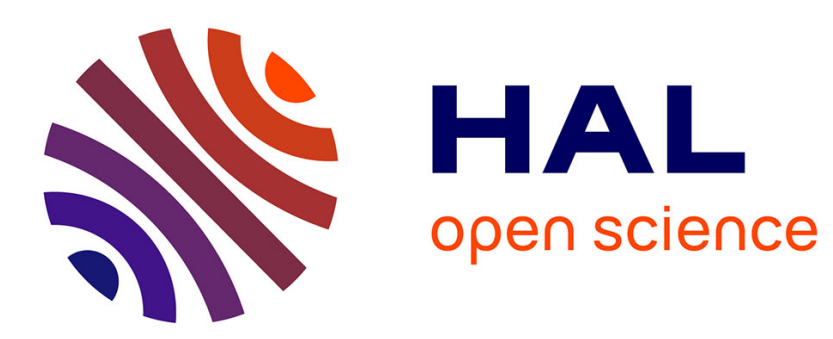

\title{
Signs of dynamical effects for Cd, Sn, Te, Xe, Ba and Sm nuclear charge radii
}

\author{
J. Libert, B. Roussière, J. Sauvage
}

\section{To cite this version:}

J. Libert, B. Roussière, J. Sauvage. Signs of dynamical effects for Cd, Sn, Te, Xe, Ba and Sm nuclear charge radii. Nuclear Physics A, 2007, 786, pp.47-70. 10.1016/j.nuclphysa.2007.01.089 . in2p300151666

\section{HAL Id: in2p3-00151666 https://hal.in2p3.fr/in2p3-00151666}

Submitted on 6 Jun 2007

HAL is a multi-disciplinary open access archive for the deposit and dissemination of scientific research documents, whether they are published or not. The documents may come from teaching and research institutions in France or abroad, or from public or private research centers.
L'archive ouverte pluridisciplinaire HAL, est destinée au dépôt et à la diffusion de documents scientifiques de niveau recherche, publiés ou non, émanant des établissements d'enseignement et de recherche français ou étrangers, des laboratoires publics ou privés. 


\title{
Signs of dynamical effects for Cd, Sn, Te, Xe, $\mathrm{Ba}$ and Sm nuclear charge radii.
}

\author{
J. Libert ${ }^{a}$, B. Roussière ${ }^{a, *}$ and J. Sauvage ${ }^{a}$ \\ anstitut de Physique Nucléaire, CNRS-IN2P3 and Université Paris Sud, F-91406 \\ Orsay Cedex, France.
}

\begin{abstract}
The experimental charge radius values along $\mathrm{Cd}, \mathrm{Sn}, \mathrm{Te}, \mathrm{Xe}, \mathrm{Ba}$ and $\mathrm{Sm}$ isotopic series have been extracted from isotope shift measurements using different methods to calibrate the electronic factor and mass shift effects. Static and dynamic charge radii have been calculated in the framework of a microscopic configuration mixing approach on the ground of Hartree-Fock-Bogoliubov solutions obtained with the D1S Gogny effective nucleon-nucleon interaction. Low-energy spectroscopic observables have also been obtained. The theoretical and experimental results are compared and discussed. It is shown that dynamical effects must be taken into account especially for $\gamma$-soft and weakly deformed nuclei.
\end{abstract}

Key words: $\mathrm{Cd}, \mathrm{Sn}, \mathrm{Te}, \mathrm{Xe}, \mathrm{Ba}$ and $\mathrm{Sm}$ isotopes, exotic nuclei, nuclear charge radius, level energy, transition probability, isotope shift, muonic atom spectroscopy, optical spectroscopy, nuclear microscopic model PACS: 21.10.Ft, 21.10.Ky, 21.10.Re, 21.60.Jz, 21.60.Ev, 23.20.-g, 36.10.Dr

\section{Introduction.}

The charge radii of nuclei, $\mathrm{r}_{c}$, and their variations along isotopic series provide key information on the nuclear matter behaviour. For example, they can reveal magic numbers and bring out changes in the nuclear deformation between either neighbouring nuclei or different states inside the same nucleus. They constitute hence one of the basic nuclear properties that any nuclear model must be able to well describe.

\footnotetext{
* Corresponding author. Tel: +33-1-69156265; fax: +33-1-69156470

Email address: roussier@ipno.in2p3.fr (B. Roussière).
} 
The most precise nuclear $r_{c}$ values have been obtained by use of the muonic atom spectroscopy method. They have been determined for almost all elements using the muon factories, but only for the stable isotopes. The most recent and precise results so obtained have been reviewed by Fricke et al. [1]. To study the $\mathrm{r}_{c}$ evolution through a large number of isotopes, we must also use the numerous results concerning the mean square charge radius changes, $\left.\delta<\mathrm{r}_{c}^{2}\right\rangle$, yielded by the optical method and choose the $r_{c}$ value of a stable mass as a reference value.

For the tin isotopes, it was recently shown that the parabolic shape of the $\mathrm{r}_{c}$ curve against the neutron number could be explained by important dynamical effects $[2-5]$. The purpose of the present work is to study the role played by these dynamical contributions as the proton number moves away from $\mathrm{N}=$ 50. The data already known [6,7] led us to choose the following elements: Cd, $\mathrm{Sn}, \mathrm{Te}, \mathrm{Xe}, \mathrm{Ba}$ and Sm.

In muonic atom spectroscopy the uncertainty on the extracted values is almost only due to the nuclear polarization corrections whereas in optical spectroscopy, to deduce the $\delta<\mathrm{r}_{c}^{2}>$ values from the isotope shift measured, one has to determine the electronic factor $\mathrm{F}$ of the optical transition as well as the part of the isotope shift due to the mass difference or mass shift MS. Various ways are currently used to get the $\mathrm{F}$ and MS values, which may provide slightly different slopes of the $\delta<\mathrm{r}_{c}^{2}>$ and $\mathrm{r}_{c}$ curves. Therefore, prior to compare nuclear model predictions with the experimental $\mathrm{r}_{c}$ curves, a very careful and critical analysis of the data has to be performed and discussed. This tricky problem will be widely treated in section 2 . The nuclear model, taking into account or not the dynamical effects, used in this work will be presented in section 3. The comparison between experimental and theoretical results will be shown and discussed in section 4. Lastly, the conclusions will be drawn in section 5 .

\section{Determination of the experimental charge radii of $\mathrm{Cd}, \mathrm{Sn}, \mathrm{Te}$, $\mathrm{Xe}, \mathrm{Ba}$ and Sm nuclei.}

Laser spectroscopy is the only experimental method giving access, from the isotope shift, to the change in the mean square charge radius through long isotopic chains including stable and unstable nuclei. The isotope shift is the displacement of the center of gravity of the hyperfine spectrum between two neighboring isotopes. When the number of neutrons varies, changes are induced in the atom, namely in the reduced mass of the nucleus plus electron system, in the correlations between the electrons and in the charge distribution inside the nucleus giving rise to the normal mass shift $\left(\delta \nu_{N M S}^{A A^{\prime}}\right)$, the specific mass shift $\left(\delta \nu_{S M S}^{A A^{\prime}}\right)$ and the field shift $\left(\delta \nu_{F S}^{A A^{\prime}}\right)$. The mass shift dominates in 
light nuclei and is small in heavy elements [6]. The normal and specific mass shifts depend in the same way on the atomic masses:

$$
\delta \nu_{M S}^{A A^{\prime}}=\delta \nu_{N M S}^{A A^{\prime}}+\delta \nu_{S M S}^{A A^{\prime}}=(\mathrm{N}+\mathrm{S}) \frac{A^{\prime}-A}{A A^{\prime}}=\mathrm{M} \frac{A^{\prime}-A}{A A^{\prime}}
$$

The normal mass shift is easy to calculate, since $\mathrm{N}$ is given by the simple formula: $\mathrm{N}=\frac{\nu_{i}}{1836.1}$ [8], where $\nu_{i}$ is the atomic transition energy. On the other hand, the specific mass shift is very difficult to calculate. For pure $s \rightarrow p$ or $\mathrm{s}^{2} \rightarrow \mathrm{sp}$ transitions, it has been shown that $|\mathrm{S}| \leq \mathrm{N}[8]$. But when $\mathrm{d}$ or $\mathrm{f}$ electrons are involved in the transition, $\mathrm{S}$ can be of the order of $10 \times \mathrm{N}$ or more.

The change in the mean square charge radius is related to the field shift by:

$$
\delta \nu_{F S}^{A A^{\prime}}=\mathrm{F} \times \lambda^{A A^{\prime}}=\mathrm{F} \times \mathrm{k} \times \delta<\mathrm{r}_{\mathrm{c}}^{2}>^{\mathrm{AA}^{\prime}},
$$

where $\mathrm{F}$ is the electronic factor of the atomic transition, $\lambda$ the nuclear parameter given by:

$$
\lambda=\delta<\mathrm{r}_{\mathrm{c}}^{2}>^{\mathrm{AA}^{\prime}}+\left(\mathrm{C}_{2} / \mathrm{C}_{1}\right) \delta<\mathrm{r}_{\mathrm{c}}^{4}>^{\mathrm{AA}^{\prime}}+\left(\mathrm{C}_{3} / \mathrm{C}_{1}\right) \delta<\mathrm{r}_{\mathrm{c}}^{6}>^{\mathrm{AA}^{\prime}}+\ldots
$$

and $\mathrm{k}$ a correcting factor calculated using the $\mathrm{C}_{n}$ Seltzer coefficients $[9,10]$. Usually the $\mathrm{F}$ factor is obtained from semi-empirical calculations using the Goudsmit-Fermi-Segré formula to evaluate the change of the electronic charge density at the nucleus. Relativistic calculations using Dirac-Fock or multiconfiguration Dirac-Fock methods are also used to determine F. Results obtained by these different methods can differ by 10 to $30 \%$ [6].

An alternative way to determine both the electronic $\mathrm{F}$ factor and the specific mass shift is by using King plot analysis [11]. In this case, the modified shifts in the optical line under study are plotted against the modified shifts in another line advisably chosen, for instance corresponding to a pure and simple configuration for which the specific mass shift can be estimated to be negligible and the F factor accurately calculated. Such a plot gives a straight line, its intercept is related to the specific mass shift and its slope equal to the ratio of the $\mathrm{F}$ factors.

The King plot analysis is also applied with data obtained from other experimental methods, such as the X-ray isotope shifts and the change in the mean square charge radius deduced from electron scattering or muonic experiments. It is worth noting that this type of experiments has been only performed on stable isotopes since it requires several tens of milligrams of target material. Using the muonic isotope shifts is not directly suitable because the difference of muonic level energy does not depend on $\delta<\mathrm{r}^{2}>$ but on $\delta<\mathrm{r}^{\mathrm{k}} \mathrm{e}^{-\alpha \mathrm{r}}>$. In the case of K-ray isotope shifts, the nuclear charge distribution investigated is the same as in optical isotope shifts but the experimental errors are too important to calibrate reliably the optical isotope shift measurements. In the same way, the accuracy obtained for the charge radius in electron scattering experiment is too low to allow the determination of the $\mathrm{F}$ factor and specific mass shift via a King plot analysis. However, electron scattering measurements give access to the radial dependence of the charge distribution. This result can be used to extract the charge radius from the very precise and model-independent Barrett radius obtained in muonic experiments. Such a method using the $\delta<\mathrm{r}_{\mathrm{c}}^{2}>$ 
values resulting from a combined analysis of muonic atom and electron scattering data has been proposed by Fricke et al. to calibrate the optical isotope shift measurements and applied for eight elements (Ca, Kr, Sr, Zr, Mo, Sm, $\mathrm{Gd}, \mathrm{Pb}$ ) [1]. However, by lack of electron scattering data, this combined analysis cannot be used for any isotope series. Nevertheless, in principle, muonic experiments give access to the better charge radius values available for the stable nuclei, in spite of the uncertainty on the calculated nuclear polarization corrections that limits their accuracy. The compilation made by Fricke et al. summarizes the results from muonic experiments performed on almost all stable nuclei and provides a consistent set of charge radius values for these stable isotopes [1]. The isotopic series we are interested in $(\mathrm{Cd}, \mathrm{Sn}, \mathrm{Te}, \mathrm{Xe}, \mathrm{Ba}$ and $\mathrm{Sm})$, located on both sides of the magic tin nuclei, have many stable isotopes $(8,10,8,9,7$ and 7 respectively). Thus, we have used the charge radius values of these stable isotopes to calibrate the isotope shift measurements performed by optical spectroscopy. Our aim is to obtain the most consistent and reliable charge radius values over long isotopic chains in order to perform very stringent comparison with theoretical calculations over a wide range of nuclei. In a first step to estimate the possible remaining uncertainty of the method, we compare the charge radius values determined using the calibration on the muonic data with those obtained with the other methods currently used. The charge radius values obtained from different methods are reported in tables 1-6 of the appendix.

\subsection{Cadmium isotopes}

We have evaluated the charge radii in three ways. Firstly we started from the $\delta\left\langle\mathrm{r}_{\mathrm{c}}^{2}\right\rangle$ values given in ref. [6]. These values have been obtained from the experimental data of ref. [12] using a factor $\mathrm{F}\left(\mathrm{F}_{S E}=3.91 \mathrm{GHz} / \mathrm{fm}^{2}\right)$ determined semi-empirically and we have deduced the charge radii taking as reference the value of the ${ }^{114} \mathrm{Cd}$ stable isotope known from the Fricke et al. compilation ref. [1]. The second estimation has consisted in determining new values for the electronic factor and the specific mass shift by doing a King plot between the optical isotope shifts measured for the stable isotopes in the $5 \mathrm{~s}^{2}$ ${ }^{1} \mathrm{~S}_{0} \rightarrow 5 \mathrm{~s} 5 \mathrm{p}{ }^{3} \mathrm{P}_{1}$ transition at $326.1 \mathrm{~nm}[12,6]$ and the charge radius values reported in ref. [1]: $\mathrm{F}_{\mu}=-4.37 \pm 0.18 \mathrm{GHz} / \mathrm{fm}^{2}$ and $\frac{\mathrm{S}}{\mathrm{N}}=2.43 \pm 0.35$ with $\mathrm{k}=$ 0.977 . Then we have used these $\mathrm{F}$ and $\frac{\mathrm{S}}{\mathrm{N}}$ values to calculate, from the ${ }^{102-120} \mathrm{Cd}$ isotope shifts of refs. [6,12], new $\delta<\mathrm{r}_{\mathrm{c}}^{2}>$ values and the corresponding charge radii taking the same reference, ${ }^{114} \mathrm{Cd}$. Lastly the comparison between the experimental isotope shifts and the $\left.\delta<\mathrm{r}_{\mathrm{c}}^{2}\right\rangle$ values extracted from ref. [1] for the stable nuclei has allowed us to determine the specific mass shift $\left(\frac{\mathrm{S}}{\mathrm{N}}\right.$ $=2.15 \pm 0.18$ ) when the value of the electronic $\mathrm{F}$ factor obtained in DiracFock calculations is used $\left(\mathrm{F}_{D F}=-4.16 \mathrm{GHz} / \mathrm{fm}^{2}\right.$, refs. $\left.[12,10]\right)$. From these 
$\delta<\mathrm{r}_{\mathrm{c}}^{2}>$ values we have obtained a third set of $\mathrm{r}_{c}$ data taking again as reference $\mathrm{r}_{c}\left({ }^{114} \mathrm{Cd}\right)$.

\subsection{Tin isotopes}

The isotope shifts have been measured from $\mathrm{A}=110$ up to 132 using the $5 \mathrm{p}^{2}{ }^{3} \mathrm{P}_{0} \rightarrow 5 \mathrm{p} 6 \mathrm{~s}{ }^{3} \mathrm{P}_{1}$ transition at $286.3 \mathrm{~nm}$ [13,5]. In ref. [13], the electronic factor has been calculated by Baird et al. [14] within a semi-empirical approach $\left(\mathrm{F}_{S E}=3.3 \pm 0.3 \mathrm{GHz} / \mathrm{fm}^{2}\right), \mathrm{a} \mathrm{k}=1$ value has been used and the specific mass shift has been evaluated from the $\delta<\mathrm{r}_{\mathrm{c}}^{2}>^{124,116}$ value obtained from isotope shift measurement of the $\mathrm{K}_{\alpha}$ transition. In ref. [5], a King plot has been performed between the optical isotope shift and the muonic $\left.\delta<\mathrm{r}_{\mathrm{c}}^{2}\right\rangle$ values measured for the stable isotopes and reported in ref. [15], this led to: $\mathrm{F}_{\mu 1}=$ $3.30 \pm 0.27 \mathrm{GHz} / \mathrm{fm}^{2}$ with $\mathrm{k}=0.975$ and $\frac{\mathrm{S}}{\mathrm{N}}=-2.33 \pm 0.35$. We have obtained a new determination of $\mathrm{F}$ and $\frac{\mathrm{S}}{\mathrm{N}}$ by performing a similar King plot analysis using the same optical isotope shift values but the muonic $\delta<\mathrm{r}_{\mathrm{c}}^{2}>$ values reported for the stable isotopes by Fricke et al:: $\mathrm{F}_{\mu 2}=2.04 \pm 0.2 \mathrm{GHz} / \mathrm{fm}^{2}$ with $\mathrm{k}=0.975$ and $\frac{\mathrm{S}}{\mathrm{N}}=-0.78 \pm 0.32$. The fourth estimation of the electronic factor, given in ref. [16] $\left(\mathrm{F}_{D F}=2.39 \pm 0.27 \mathrm{GHz} / \mathrm{fm}^{2}\right)$, has been deduced from a King plot with results from another optical transition for which $\mathrm{F}$ was obtained using Dirac-Fock calculations. The specific mass shift has been evaluated from the $\delta<\mathrm{r}_{\mathrm{c}}^{2}>^{124,116}$ value measured from the $\mathrm{K}_{\alpha}$-transition isotope shift $\left(\frac{\mathrm{S}}{\mathrm{N}}=\right.$ -1.04 ) and a $\mathrm{k}=1$ value has been used. From these sets of $\delta<\mathrm{r}_{\mathrm{c}}^{2}>$ data, we have calculated the charge radii taking as reference the value reported in ref. [1] for ${ }^{120} \mathrm{Sn}$, except for the $\delta<\mathrm{r}_{\mathrm{c}}^{2}>$ values obtained from $\mathrm{F}_{\mu 1}$. In this case, for a consistence purpose, we have used the $\mathrm{r}_{c}$ value given in ref. [15] for ${ }^{120} \mathrm{Sn}$.

\subsection{Tellurium isotopes}

The neutron-rich tellurium isotopes have been recently studied by laser spectroscopy using the $5 \mathrm{p}^{4}{ }^{3} \mathrm{P}_{2} \rightarrow 5 \mathrm{p}^{3} 6 \mathrm{~s}^{3} \mathrm{~S}_{1}$ transition at $214.3 \mathrm{~nm}[17,18]$. In this work the electronic factor and the specific mass shift have been determined by performing a King plot between the preliminary optical isotope shifts and the $\delta<\mathrm{r}_{c}^{2}>$ values obtained from muonic experiment [19]: $\mathrm{F}_{\mu 1}=4.66 \pm 0.86$ $\mathrm{GHz} / \mathrm{fm}^{2}$ with $\mathrm{k}=0.974$ and $\frac{\mathrm{S}}{\mathrm{N}}=-1.89 \pm 0.33$. Starting from the same optical isotope shift values we have done a second determination of the electronic factor and specific mass effect using the muonic $\delta<\mathrm{r}_{c}^{2}>$ values reported in ref. [1]: $\mathrm{F}_{\mu 2}=3.78 \pm 0.48 \mathrm{GHz} / \mathrm{fm}^{2}$ with $\mathrm{k}=0.974$ and $\frac{\mathrm{S}}{\mathrm{N}}=-1.73 \pm 0.37$. Then we have calculated the resulting charge radii taking as reference the ${ }^{130} \mathrm{Te}$ charge radius in the first case from [19] and in the second case from ref. [1]. 


\subsection{Xenon isotopes}

The optical isotope shifts have been measured from $\mathrm{A}=116$ up to 146 in the $5 \mathrm{p}^{5}$ 6s $\left[\frac{3}{2}\right]_{2} \rightarrow 5 \mathrm{p}^{5} 6 \mathrm{p}\left[\frac{3}{2}\right]_{2}$ transition at $823.2 \mathrm{~nm}[20]$. The $\delta<\mathrm{r}_{c}^{2}>$ values have been obtained using the electronic factor calculated in semi-empirical approach $\left(\mathrm{F}_{S E}=-2.32 \mathrm{GHz} / \mathrm{fm}^{2}[6]\right)$ and assuming the specific mass shift to be zero with an error of \pm the normal mass shift. Using the $\delta<\mathrm{r}_{c}^{2}>$ given in ref. [20] and taking as reference the ${ }^{136} \mathrm{Xe}$ charge radius reported in ref. [1] we have calculated the charge radii from ${ }^{116} \mathrm{Xe}$ up to ${ }^{146} \mathrm{Xe}$. We have determined new values for the electronic factor and the specific mass shift by doing a King plot of the optical isotope shifts [20] versus the $\delta\left\langle\mathrm{r}_{c}^{2}\right\rangle$ values reported in ref. [1] : $\mathrm{F}_{\mu}=-1.56 \pm 0.11 \mathrm{GHz} / \mathrm{fm}^{2}$ with $\mathrm{k}=0.972$ and $\frac{\mathrm{S}}{\mathrm{N}}=0.192 \pm 0.266$. Then we have calculated the corresponding $\delta<\mathrm{r}_{c}^{2}>$ from $\mathrm{A}=116$ up to 146 and deduced the charge radii taking again as reference the ${ }^{136} \mathrm{Xe}$ charge radius value reported in ref. [1].

\subsection{Barium isotopes}

The optical isotope shifts have been measured from $\mathrm{A}=120$ up to $\mathrm{A}=148$ using two transitions: the $6 \mathrm{~s}^{2}{ }^{1} \mathrm{~S}_{0} \rightarrow 6 \mathrm{~s} 6 \mathrm{~s}^{1} \mathrm{P}_{1}$ transition at $553.6 \mathrm{~nm}$ and the BaII D2 line at $455.4 \mathrm{~nm}$ (see the laser spectroscopy studies listed in ref. [6]). In the following we will concentrate on the $553.6 \mathrm{~nm}$ transition because, in this case, the electronic factor has been obtained not only from semi-empirical approach but also in multiconfiguration Dirac-Fock calculations [10]. We have used three different methods to estimate the Ba charge radii. Firstly starting with the $\delta<\mathrm{r}_{c}^{2}>$ values from ref. [6], we have calculated the charge radii taking as reference the $\mathrm{r}_{c}$ value reported in ref. [1] for ${ }^{138} \mathrm{Ba}$. The $\left.\delta<\mathrm{r}_{c}^{2}\right\rangle$ values given in ref. [6] have been obtained with the following semi-empirical electronic factor and specific mass shift: $\mathrm{F}_{S E}=-3.929 \mathrm{GHz} / \mathrm{fm}^{2}$ and $\frac{\mathrm{S}}{\mathrm{N}}=0$. Secondly we have performed a King plot using the optical isotope shifts measured for the stable isotopes [22] and the charge radius values obtained from muonic experiments and reported in refs. [21] or [1], this led using $\mathrm{k}=0.97$ to $\mathrm{F}_{\mu 1}=-3.163 \pm 0.144$ $\mathrm{GHz} / \mathrm{fm}^{2}$ and $\frac{\mathrm{S}}{\mathrm{N}}=-0.707 \pm 0.109$ or $\mathrm{F}_{\mu 2}=-3.897 \pm 0.189 \mathrm{GHz} / \mathrm{fm}^{2}$ and $\frac{\mathrm{S}}{\mathrm{N}}=$ $2.649 \pm 0.27$, respectively. Using the $\delta \nu$ values measured from $\mathrm{A}=122$ to $\mathrm{A}=$ 146 in the $553.6 \mathrm{~nm}$ transition [22,23], we have deduced the $\delta<\mathrm{r}_{c}^{2}>$ values corresponding to these electronic factors and specific mass shift effects and then the $r_{c}$ values taking as reference the ${ }^{138}$ Ba charge radius [21,1]. Finally we have evaluated the specific mass shift corresponding to the electronic factor obtained in multiconfiguration Dirac-Fock calculations $\left(\mathrm{F}_{M C D F}=-2.996\right.$ $\left.\mathrm{GHz} / \mathrm{fm}^{2}[10]\right)$ by comparing the $\delta \nu$ values and the muonic $\delta<\mathrm{r}_{c}^{2}>$ values for the stable isotopes: $\frac{\mathrm{S}}{\mathrm{N}}=0.1 \pm 1.1$. A new set of $\mathrm{r}_{c}$ data has been obtained 
taking as reference the charge radius given for ${ }^{138} \mathrm{Ba}$ in ref. [1].

\subsection{Samarium isotopes}

Many laser spectroscopy studies have been performed in the samarium isotopes, they have been summed up in ref. [6]. The optical isotope shifts have been measured from $\mathrm{A}=138$ to 145 using the $4 \mathrm{f}^{6} 6 \mathrm{~s}^{2}{ }^{7} \mathrm{~F}_{2} \rightarrow 4 \mathrm{f}^{6} 6 \mathrm{~s} 6 \mathrm{p}^{5} \mathrm{G}_{2}$ transition at $600.42 \mathrm{~nm}[24]$ and from $\mathrm{A}=146$ to 154 using the $4 \mathrm{f}^{6}{ }^{6} \mathrm{~s}^{2}{ }^{7} \mathrm{~F}_{1} \rightarrow 4 \mathrm{f}^{6}$ 6s $6 \mathrm{p}^{7} \mathrm{~F}_{0}$ transition at $570.68 \mathrm{~nm}$ [25]. The isotope shifts of the stable isotopes have been performed extensively, in 15 lines of the SmI spectrum including the $570.68 \mathrm{~nm}$ [26]. Thus for this transition we could carry out a King plot analysis using the optical shift and the muonic $\delta\left\langle\mathrm{r}_{c}^{2}\right\rangle$ values given either in refs. [27,28] or in ref.[1], this led, using $\mathrm{k}=0.963$, to $\mathrm{F}_{\mu 1}=-2.82 \pm 0.14$ $\mathrm{GHz} / \mathrm{fm}^{2}$ and $\frac{\mathrm{S}}{\mathrm{N}}=-16.37 \pm 1.84$ or $\mathrm{F}_{\mu 2}=-2.48 \pm 0.23 \mathrm{GHz} / \mathrm{fm}^{2}$ and $\frac{\mathrm{S}}{\mathrm{N}}=$ $-18.79 \pm 3.01$, respectively. Moreover, for this transition, Fricke et al. have performed a combined analysis of the optical isotope shifts and of the data obtained from muonic atom and electron scattering experiments, which led to $\mathrm{F}_{\mu e^{-}}=-3.86 \pm 0.86 \mathrm{GHz} / \mathrm{fm}^{2}$ and $\frac{\mathrm{S}}{\mathrm{N}}=0.4 \pm 1.3$. In the $600.42 \mathrm{~nm}$ transition, only the isotope shift of the ${ }^{144,147} \mathrm{Sm}$ stable nuclei has been measured, which is insufficient for a calibration using the muonic data. Finally the charge radius values in Sm have been calculated in four ways. Firstly, we have used the semi-empirically determined $\delta\left\langle\mathrm{r}_{c}^{2}\right\rangle$ values given in ref. [6] for $\mathrm{A}=138-154$ and taken as reference the charge radius of ${ }^{144} \mathrm{Sm}$ reported in ref. [1]. For $\mathrm{A}=$ 144-154, we have evaluated the charge radii from the isotope shifts measured

in the $570.68 \mathrm{~nm}$ transition and the $\mathrm{F}$ and $\frac{\mathrm{S}}{\mathrm{N}}$ values resulting from the King plots performed using either the muonic atom data or the combined analysis. The charge radius of the reference nucleus, ${ }^{144} \mathrm{Sm}$, was taken from ref. [1], except when the muonic data used for the King plot were those of refs. [27,28]. In this case, in order to be consistent we have used the ${ }^{144} \mathrm{Sm} \mathrm{r}_{c}$ value reported in ref. [27].

The charge radius values obtained as indicated above are presented in fig. 1 . Before $\mathrm{N}=82$ the charge radii obtained using semi-empirical $\mathrm{F}$ factors are systematically higher than those using the $\mathrm{F}$ factors determined by a King plot analysis between the optical isotope shifts and the muonic $\delta<\mathrm{r}_{c}^{2}>$ values for the stable isotopes. The contrary is observed after $\mathrm{N}=82$. However the scattering of the values is not very important and one can wonder whether the error bars are underestimated. For instance, in case of $\mathrm{Cd}$, the possible systematic error on $\delta<\mathrm{r}_{c}^{2}>$ due to the uncertainty in the specific mass shift effect and $\mathrm{F}$ factor has been estimated and is indicated in ref. [12]. Taking into account these systematic errors results in multiplying by a factor of 5 the error bars in the charge radius as compared with the ones reported in table 1 column 3, and makes this radius value set consistent with the two others 
drawn in figure 1.

It is in the Xe isotope series that the larger differences are observed between the charge radius sets. But the information on the laser-spectroscopy and muonic-atom experiment results is scanty; in particular the charge radius values from muonic-atom experiment are only available in the compilation of Fricke et al. [1]. However we can see in fig. 1 that the differences in charge radii between the two data sets are less than or around one per cent. On the other hand, analysing, for different $\mathrm{N}$ values the evolution of the charge radius as a function of $\mathrm{Z}$, one can conclude that the true charge radius values lie somewhere between the values labelled $\mathrm{F}_{S E}$ and those labelled $\mathrm{F}_{\mu}$.

For $\mathrm{Sn}, \mathrm{Te}$, Ba and $\mathrm{Sm}$, two charge radius sets are available for the stable isotopes from muonic-atom experiment, leading to two determinations of the $\mathrm{F}$ factor and specific mass shift effect. One can note that there is a compensation between these two parameters since the charge radius values obtained over the whole isotope series remain very similar even when the $\mathrm{F}$ and $\frac{\mathrm{S}}{\mathrm{N}}$ factors are significantly different.

In the following, for the comparison with theory, in a first step we will use the charge radius values labelled $\mathrm{F}_{\mu}$ or $\mathrm{F}_{\mu 2}$ in fig. 1 or tables 1-6. They present the advantage of consistency since they have been obtained applying the same method for the determination of the $\mathrm{F}$ factor and specific mass shift effect, using in all cases the charge radii reported for the stable isotopes in the compilation by Fricke et al. [1]. For ${ }^{138-143} \mathrm{Sm}$ that have not been studied with the same optical transition than the heavier isotopes, we will use the charge radius values from the data set labelled $\mathrm{F}_{S E}$. Finally it is worth noting that the table of nuclear charge radii published by Angeli [29] supports quite well the data sets that we have chosen to compare with the results of calculations.

\section{Microscopic evaluation of nuclear charge radii and spectroscopic properties.}

A systematic investigation of $\mathrm{Cd}, \mathrm{Sn}, \mathrm{Te}, \mathrm{Xe}, \mathrm{Ba}$ and $\mathrm{Sm}$ isotopic chains has been performed on the ground of Hartree-Fock-Bogoliubov solutions deduced under triaxial symmetries from the D1S Gogny effective nucleon-nucleon interaction [30-32]. At first step for each nucleus, constrained HFB (CHFB) solutions are calculated by expansion of single particle states on a triaxial harmonic oscillator basis. Involved numerical methods and codes for this purpose are those described in ref. [33]. CHFB solutions are mapped against axial $q_{0}=\left\langle\widehat{Q}_{20}\right\rangle$ and triaxial $q_{2}=\left\langle\widehat{Q}_{22}\right\rangle$ components of the mass quadrupole moment. This mapping is as it is usual rewritten in term of polar Bohr's variables: 


$$
\begin{aligned}
& \beta=\frac{\sqrt{5 \pi}}{3} \frac{\sqrt{q_{0}^{2}+3 q_{2}^{2}}}{A\left(A^{\frac{1}{3}} r_{0}\right)^{2}} \\
& \gamma=\arctan \sqrt{3} \frac{q_{2}}{q_{0}}
\end{aligned}
$$

or their usual cartesian counterparts $\left(a_{0}=\beta \cos \gamma, a_{2}=\beta \sin \gamma\right)$. In the eq. 1 which connects scaled collective variables $(\beta, \gamma)$ with quadrupole moments, the standard liquid drop expression for the mean radius is employed with $r_{0}=1.2 \mathrm{fm}, A$ being the particle number.

As known, due to symmetry invariances, this mapping can be restricted to the first sextant $\left\{0 \leqslant \beta<+\infty ; 0 \leqslant \gamma \leqslant \frac{\pi}{3}\right\}$. In practice, the CHFB calculations are performed on a lattice with typically 80 mesh points in the first sextant with $0 \leqslant \beta \leqslant \beta_{c}$ using the cutoff value $\beta_{c}=0.65$ which will secure, in present case, the evanescent character of collective wave functions (see hereafter).

In each mesh point $(\beta, \gamma)$, the neutron $\rho_{n}^{\beta \gamma}(\vec{r})$ and proton $\rho_{p}^{\beta \gamma}(\vec{r})$ densities associated with the CHFB solution $\phi^{\beta \gamma}$ give, in particular, the value of neutron and proton point mean square radius by:

$$
\left\langle r_{k}^{\beta \gamma}\right\rangle^{2}=\frac{1}{N_{k}} \int d^{3} r \rho_{k}^{\beta \gamma}(\vec{r}) r^{2} \text { with } k=n \text { or } p
$$

where $N_{p}$ and $N_{n}$ are the proton and neutron numbers. According to [31] and [34], charge radii are then written in fm units as:

$$
\begin{aligned}
r_{c}^{\beta \gamma} & =\sqrt{\left\langle r_{p}^{\beta \gamma}\right\rangle^{2}+f_{c o n v}^{\beta \gamma}+\varepsilon_{n}} \\
\text { with } f_{c o n v}^{\beta \gamma} & =\frac{3}{2}\left(B^{2}-a\right) \text { and } \varepsilon_{n}=-0.12 \frac{N_{n}}{N_{p}}\left(\mathrm{fm}^{2}\right)
\end{aligned}
$$

In former expressions, $f_{\text {conv }}^{\beta \gamma}$ carries a proton form factor term $(B=0.65$ $\mathrm{fm}$ ) and a correction $a$ due to center of mass motion. This correction, known to be small, is evaluated assuming pure harmonic oscillator wave functions with $a=\hbar /\left(m \omega_{\beta \gamma} A\right)$ where $m$ is the nucleonic mass $\left(\frac{\hbar^{2}}{m}=41.47 \mathrm{MeV} \mathrm{fm}{ }^{2}\right)$ and where $\omega_{\beta \gamma}$ is the oscillator constant value (in $\mathrm{MeV} / \hbar$ ) chosen in each mesh point to minimize the CHFB energy. Finally, $\varepsilon_{n}$ is a standard expression of the contribution to charge radii associated with the neutron electromagnetic properties.

The HFB state - i.e. the solution $\phi_{\beta_{m} \gamma_{m}}$ at the minimum of total energy which arises at deformation $(\beta, \gamma)=\left(\beta_{m}, \gamma_{m}\right)$ - defines what will be called hereafter 
the "static" properties and, in particular, the charge radius:

$$
r_{c}^{H F B}=r_{c}^{\beta_{m} \gamma_{m}}
$$

At this step, we have applied a configuration mixing approach in the space spanned by the CHFB states $\phi^{\beta \gamma}$ and their rotation transforms. The general frame is the Generator Coordinate Method (GCM) under Gaussian Overlap Approximation (GOA) for the complete quadrupole collective space i.e. for axial and triaxial quadrupole vibrations together with rotation treated in this frame on the same footing. Similar approach has been applied to the description of low-energy spectroscopy in various regions of the nuclear chart, and in particular in [35] for light $\mathrm{Hg}$ isotopes. Present approach is identical in its principles to those implemented for the description of the Normal-Super Deformation (ND-SD) shape coexistence in mercury-lead and actinide regions $[36,37]$. Technical details can be found in ref. [36] and refs. therein. This approach has been shown to be able to reproduce main trends for low-energy collective levels in deformed and transitional nuclei. However, as well known, the considered collective space is not well adapted to rigid spherical (magic and particularly doubly magic) system, and some evidence of this weakness has thus to be expected in some nuclei of our panel. Here, we will only recall the main steps which are involved in the method.

i) Under the GOA approximation, the variational GCM Hill-Wheeler equation in the five components of the quadrupole tensor $\left\{q_{2 m}\right\}$ can be re-written in the intrinsic system under a Schrödinger form i.e. an Hamiltonian $\mathcal{H}^{\text {coll }}$ which takes the form of the so-called full quadrupole collective Bohr Hamiltonian in $\beta, \gamma$, and Euler angles $\Omega=(\theta, \varphi, \psi)$. $\mathcal{H}^{\text {coll }}$ has analytically the same form as the Bohr Hamiltonian considered and numerically solved for the first time in the pioneering work of ref. [38].

ii) The Hamiltonian $\mathcal{H}^{\text {coll }}$ involves a kinetic term made with six functions of the deformation, namely the three moments of inertia $\left\{\mathcal{J}_{k}(\beta, \gamma)\right\}_{k}$ (where $k$ refers to the principal axes $(k=1,2$ and 3$))$ and three mass parameters allowing to built the symmetric vibrational kinetic tensor $\left\{B_{\alpha \alpha^{\prime}}(\beta, \gamma)\right\}_{\alpha, \alpha^{\prime}=\{\beta, \gamma\}}$ associated with vibrations in $\beta$ and $\gamma$ directions and their coupling. These ingredients are calculated for each CHFB solution $\phi^{\beta \gamma}$ using the perturbative approach of motion and linear response theory driving to the so-called Inglis-Belyaev formulas. However, previous works using self-consistent cranking calculations have driven us to introduce a simple but realistic overall scaling factor on $\mathcal{J}_{k}$ functions to take into account in a simple way the effect of rotation on the nuclear field (the so-called dynamical Thouless-Valatin contribution).

Under GCM-GOA hypotheses, the potential term $V=E^{C H F B}-E_{z p}$ of $\mathcal{H}^{\text {coll }}$ carries rotational and vibrational Zero Point Energy contributions which are also evaluated by Inglis-Belyaev cranking series belonging to the linear response theory. Finally, we have used here as it is the case for instance in [36] 
and [37]:

$$
\begin{aligned}
\mathcal{J}_{k}(\beta, \gamma) & =1.32 * \mathcal{J}_{k}^{\text {Inglis-Belyaev }}(\beta, \gamma), k=1,2,3 \\
B_{\alpha \alpha^{\prime}}(\beta, \gamma) & =B_{\alpha \alpha^{\prime}}^{\text {Inglis-Belyaev }}(\beta, \gamma), \alpha, \alpha^{\prime}=\{\beta, \gamma\} \\
V(\beta, \gamma) & =E^{C H F B}(\beta, \gamma)-E_{z p}^{\text {Inglis-Belyaev }}(\beta, \gamma)
\end{aligned}
$$

iii) The Schrödinger equation is solved numerically using expansion techniques similar to those explained in great details in [36]. Deduced eigen energies, $E^{I, n}$ ordered by quantum number $n$ in each block of good angular momentum $I$, are associated with eigen states taking the form of normalized combinations:

$$
\Psi^{I, n}=\sum_{\text {Keven } \geqslant 0} A_{K}^{I, n}(\beta, \gamma) \varphi_{M K}^{I}(\Omega)
$$

which carries spreading in deformation variables and K-mixing. In eq. 7, $\varphi_{M K}^{I}(\Omega)$ refers to the standard combination (see. e.g. [38]) of Wigner rotation matrices for angular momentum $I$ and its projections $\pm K$ and $M$ on the third axis in intrinsic and laboratory frame, respectively.

Here we focus our interest on properties of the ground states. For this state $(I=K=0, n=1)$, the density of probability in collective space $\rho^{0,1}(\beta, \gamma)$ is given by:

$$
\rho^{0,1}(\beta, \gamma)=\left|A_{0}^{0,1}(\beta, \gamma)\right|^{2} \mu(\beta, \gamma)
$$

where $\mu$ is the metric involving moments of inertia and mass parameters, in such a way that for the normalized state, one has:

$$
\int_{\text {sextant }} \rho^{0,1}(\beta, \gamma) \beta d \beta d \gamma=1
$$

The associated charge radius, named in what follows "dynamic" or GCM-GOA charge radius writes:

$$
r_{c}^{G C M-G O A}=\int_{\text {sextant }} \rho^{0,1}(\beta, \gamma) r_{c}^{\beta \gamma} \beta d \beta d \gamma
$$

where $r_{c}^{\beta \gamma}$ is the local CHFB value defined in eq. 3. Other ground-state properties as $\langle\beta\rangle$ and $\langle\gamma\rangle$ are deduced in the same way through the knowledge of the density $\rho^{0,1}(\beta, \gamma)$. 
In the panel of nuclei under study, we can isolate three types of typical behavior in the collective space. Potentials Energy Surfaces (PES) and deduced densities $\rho^{0,1}$ for rigid or very rigid spherical nuclei are displayed in fig. 2 for ${ }^{98,130} \mathrm{Cd}$ nuclei taken as examples. Similar information for a soft mid-shell nuclei $\left({ }^{116} \mathrm{Sn}\right)$ is shown in fig. 3, whereas a prolate well-deformed behavior is displayed in fig. 4 through the ${ }^{126} \mathrm{Ba}$ and ${ }^{154} \mathrm{Sm}$ cases.

Hereafter and in the discussion of section 4, we will also refer to reduced E2 transition probability values calculated for the first transition in the ground state (gs) band, namely $0_{g s}^{+} \rightarrow 2_{g s}^{+}$. These calculations have been performed under simple hypothesis of uniform charge distribution in collective model following [38]. That is known to be quite reliable as long as a shape transition does not occur between the initial and the final states. Qualitatively, one can retain that the microscopic charge quadrupole operator, which is non local in the present collective space, can be replaced by the corresponding local collective model operator as long as densities in the initial and final states are high in the same area of the collective space. This is clearly the case for nuclei under study, and, in particular for the transition $0_{g s}^{+} \rightarrow 2_{g s}^{+}$.

To be more explicit on this point, collective model assumption of ref. [38], means that the considered charge quadrupole operator acting in the collective space is taken simply as the two component operator:

$$
\begin{aligned}
\widehat{Q_{0}} & =\frac{3}{4 \pi} Z\left(r_{0} A^{\frac{1}{3}}\right)^{2} \beta \cos \gamma \\
\widehat{Q}_{2^{\prime}} & =\frac{3}{4 \pi} Z\left(r_{0} A^{\frac{1}{3}}\right)^{2} \beta \sin \gamma
\end{aligned}
$$

with $\mathrm{r}_{0}=1.2 \mathrm{fm}$.

Under these hypotheses, the reduced E2 transition probability between an initial $(I, n)$ and and a final $\left(I^{\prime}, n^{\prime}\right)$ state of our spectrum, writes:

$$
B\left(E 2 ;(I, n) \rightarrow\left(I^{\prime}, n^{\prime}\right)\right)=\frac{1}{(2 I+1)}\left|\left\langle I, n\|\mathcal{M}(E 2)\| I^{\prime} n^{\prime}\right\rangle\right|^{2}
$$

where the reduced matrix element, for transitions $(0, n) \rightarrow\left(2, n^{\prime}\right)$ under present scope writes after evaluating geometrical coefficients:

$$
\left\langle 0, n\|\mathcal{M}(E 2)\| 2, n^{\prime}\right\rangle=\left\langle A_{0}^{0, n}\left|\widehat{Q_{0}}\right| A_{0}^{2, n^{\prime}}\right\rangle+\left\langle A_{0}^{0, n}\left|\widehat{Q_{2^{\prime}}}\right| A_{2}^{2, n^{\prime}}\right\rangle
$$

with

$$
\left\langle A_{K}^{I, n}\left|\widehat{Q_{m}}\right| A_{K^{\prime}}^{I^{\prime}, n^{\prime}}\right\rangle=\int_{\text {sextant }} A_{K}^{I, n}(\beta, \gamma) \widehat{Q_{m}} A_{K^{\prime}}^{I, n^{\prime}}(\beta, \gamma) \mu(\beta, \gamma) \beta d \beta d \gamma
$$


This paper is focused on measurement and evaluation of charge radii. To control the validity of the present GCM-GOA approach, it is however of some interest to see some results obtained in this frame for other observables as excitation energy of first levels in the ground state band $\left(2_{g s}^{+}, 4_{g s}^{+}\right)$and these

E2 transition rates $\left(0_{g s}^{+} \rightarrow 2_{g s}^{+}\right)$discussed above. For all isotopic chains under study, these results are drawn in fig. 5. The main trends are satisfactorily reproduced for these quantities. Main discrepancies appear for magic and doubly magic nuclei. The only case where the B(E2) is over-evaluated do correspond to the magic $\mathrm{N}=50 \mathrm{Sn}$ nuclei. This overestimation of the collectivity corresponds to a certain inadequation of the considered space as already mentioned. The very strong effect on excitation energies due to the $\mathrm{N}=82$ shell closure is often missed by the calculation. It should be noticed, however, that such approaches under quasiparticle hypothesis, manage with a spreading in the number of particles. It could not be very surprising to see a smoothing on excitation energies as function of $\mathrm{N}$ in the vicinity of the violent $\mathrm{N}=82$ shell closure. The phenomenon is strong and makes the systems with $\mathrm{N} \pm 2$ quite different from the system with $\mathrm{N}$ neutrons. Keeping in mind that we have to take care around shell and doubly shell closures, the overall agreement with experimental data which has been shown demonstrates that the present theoretical description provides reliable gs wave functions and therefore a convenient ground to study in more detail the question of charge radii.

\section{Discussion.}

The static and dynamic root mean square charge radii calculated within the theoretical approach described above are displayed in fig 6. Following the analysis of section 2, they are compared in this figure with the $\mathrm{r}_{c}$ values we deduced from the optical isotope shift measurements by a King plot analysis performed using the $\mathrm{r}_{c}$ values known for the stable isotopes either from muonic atom experiments or from a combined analysis.

For the Cd, Sn, Te and Sm isotopic series, we must note that the best agreement is clearly obtained between the experimental data and the dynamic theoretical predictions. However for the nuclei having a neutron number very close to a magic one $(\mathrm{N}=50$ or 82$)$, the agreement can become better with the static calculations; this is the case for the doubly magic ${ }^{132} \mathrm{Sn}$, magic ${ }^{134} \mathrm{Te}$, Cd with $\mathrm{N}<58$ and Sm with $76<\mathrm{N}<84$.

On the other hand, for the Xe and Ba isotopic series, some of the $\mathrm{r}_{c}$ values are rather in best agreement with these given by the static predictions whereas the curves have a smooth behaviour similar to this predicted by the dynamic calculations. One can note, in particular, that the jumps found in the $\mathrm{r}_{c}$ static 
curves never exist in the experimental ones. The jumps happen close to the neutron mid-shell $\mathrm{N}=66$; namely between $\mathrm{N}=64$ and 66 for the Te nuclei and between $\mathrm{N}=70$ and 72 for the Xe and Ba ones. The potential energy surfaces and the corresponding densities of ${ }^{116,118} \mathrm{Te}(\mathrm{N}=64$ and $\mathrm{N}=66)$ and ${ }^{124,126} \mathrm{Xe}(\mathrm{N}=70$ and $\mathrm{N}=72)$ are shown in figs. 7 and 8 . These potential energy surfaces are smooth with a very large $\gamma$ valley and without any deep well, which clearly indicates that the four nuclei are $\gamma$-soft. In such a case, the HFB solution localized at the minimum is a poor description of the system which strongly spreads out on the deformation space. This means that the deduced static deformation is then not valuable. For example, the potential energy surfaces of ${ }^{116} \mathrm{Te}$ and ${ }^{118} \mathrm{Te}$ are very similar but a small minimum is visible at $\beta=0.35$ for ${ }^{116} \mathrm{Te}$ whereas no minimum appears for ${ }^{118} \mathrm{Te}$. This very small difference could explain the static $r_{c}$ curve jump that is found between ${ }^{116} \mathrm{Te}$ and ${ }^{118} \mathrm{Te}$ because of the obvious strong influence of the deformation on the $\mathrm{r}_{c}$ value. The static and dynamic deformation parameters are shown in fig. 9. We can see that the static deformation parameter $\beta=0.35$ for ${ }^{116} \mathrm{Te}$ is large while it is only $\beta=0.17$ for ${ }^{118} \mathrm{Te}$. In the same way the static $\mathrm{r}_{c}$ curve jumps calculated at $\mathrm{N}=70$ for the $\mathrm{Xe}$ and $\mathrm{Ba}$ isotopic series are due to sudden changes of the deformation parameters (see fig. 9). We can conclude that the local irregularities found for the $\mathrm{r}_{c}$ curves with the static calculations are artefacts that can come out for a soft nucleus for which the potential energy surface is very smooth.

Such artefacts do not exist in dynamic calculations since the density is determined taking into account the shape of the whole potential energy surface. Thus, for example, the densities calculated for ${ }^{116} \mathrm{Te}$ and ${ }^{118} \mathrm{Te}$ are very similar, they spread out on a large part of the collective space, from $\beta=0.1$ to 0.4. In such cases, good predictions can only be obtained by use of dynamical calculations, taking into account long range correlations. So, except for nuclei situated very close to the magic numbers, the dynamic approach is expected to provide the best description.

Now, it remains to understand why the static $\mathrm{r}_{c}$ values seem to be in a better agreement with the experimental $\mathrm{r}_{c}$ values than the dynamic ones for the $\mathrm{Xe}$ and $\mathrm{Ba}$ isotopic series. Is this due to the calculation quality or to the experimental determination? To seek to answer the first question, it is possible to estimate the calculation quality by having a look again at the fig. 5 where some other experimental and predicted spectroscopic properties $(\mathrm{B}(\mathrm{E} 2)$, $\mathrm{E}(2+), \mathrm{E}(4+) / \mathrm{E}(2+))$ are compared. The agreement between predicted and experimental results is at least as good for the $\mathrm{Xe}$ and $\mathrm{Ba}$ isotopic series as for the other ones. The agreement is even particularly excellent for the $\mathrm{Ba}$ nuclei. The calculation quality does not seem, hence, to be called in question for $\mathrm{Xe}$ and $\mathrm{Ba}$ nuclei. As for the second question, we have to remind that the larger difference between the various experimental determinations has been obtained for the Xe and Ba isotopic series (see fig. 1). Moreover, some rea- 
sons of uncertainty in the $\mathrm{r}_{c}$ determination in the Xe isotopic series have been stressed in section 2. So, for Xe and Ba nuclei, we compare in fig. 10 the $\mathrm{r}_{c}$ values obtained with dynamic calculations with the two sets of data given by the semi-empirical method $\left(\mathrm{F}_{S E}\right)$ and the King plot determination $\left(\mathrm{F}_{\mu 2}\right)$ using the muonic atom results. The best agreement is obviously obtained with the data set semi-empirically determined. It is worth noting that the muonic $\mathrm{r}_{c}$ values of the stable isotopes (reported as open triangle in fig. 6) used to perform the King plot analysis, are all located close to the magic neutron number $\mathrm{N}=82$ where the $\mathrm{r}_{c}$ values are almost constant and consequently where the mean square charge radius changes are very small. In these particular conditions the $\mathrm{F}$ value can be not enough precisely determined to get the right $\mathrm{r}_{c}$ values for the nuclei located far away from the magic number. Moreover, the muonic $r_{c}$ values reported in the Fricke table (ref. [1]) for the stable Xe and Ba nuclei have been corrected for nuclear polarization effects assuming a spherical nuclear shape whereas for the lightest isotopes a deformation already exists. This approximation could also contribute to increase the uncertainty on the $\mathrm{F}$ value. All of these facts show the limit of the method. For these critical cases it is very clear that a combined analysis as that used for the Sm nuclei, is absolutely necessary to get valuable results to be compared with theoretical predictions.

\section{Conclusion.}

In this work, the $\mathrm{r}_{c}$ values along the $\mathrm{Cd}$, Sn, Te, Xe, Ba and Sm isotopic series have been obtained using mainly the muonic atom and optical spectroscopy results. Among the various methods employed to determine the electronic factor $\mathrm{F}$ and the Specific over Normal mass shift S/N, the King plot analysis using the $\mathrm{r}_{c}$ values extracted for the stable isotopes from muonic atom experiments leads as expected to the most accurate $r_{c}$ values. However, this method seems to reach its limits in cases where the reference stable isotopes are close to a magic number and drive therefore a low slope of the $r_{c}$ curve as function of the neutron number. This has been shown in Xe and Ba isotopic chains. In such cases, a combined analysis would be essential but is unfortunately unavailable up to now.

The excellent agreement found between the experimental data and dynamical GCM-GOA calculations for Cd Sn Te isotopes has shown that a theoretical approach must take into account long range correlations especially for $\gamma$-soft and weakly deformed nuclei. Of course in cases of well deformed nuclei, as $\mathrm{Sm}$, the static and dynamic deformations are similar and lead to almost the same $r_{c}$ values. In all the isotopic series under study, the low-energy spectroscopy is well reproduced by GCM-GOA calculations. In particular, this is the case for the Xe and Ba chains. In these nuclei, the GCM-GOA $r_{c}$ values 
are thus expected to be also in good agreement with the experimental values. The fact that the dynamical charge radii are in better agreement with the experimental $\mathrm{r}_{c}$ values obtained using the semi-empirical $\mathrm{F}_{S E}$ factor than with those calculated using the muonic $\mathrm{F}_{\mu}$ factor supports the limitation of the $\mathrm{F}_{\mu}$ determinations evocated above for the Xe and Ba nuclei. 


\section{Appendix.}

Table 1

Charge radii (in $\mathrm{fm}$ ) for $\mathrm{Cd}$ isotopes

\begin{tabular}{|c|c|c|c|c|c|c|}
\hline $\mathrm{A}$ & $\mathrm{N}$ & $\mathrm{F}_{S E^{a}}{ }^{a}$ & $\mathrm{~F}_{\mu}{ }^{b)}$ & $\mathrm{F}_{D F}{ }^{c)}$ & HFB & GCM-GOA \\
\hline 98 & 50 & & & & 4.4354 & 4.4614 \\
100 & 52 & & & 4.4487 & 4.4684 \\
102 & 54 & $4.5022 \pm 0.0058$ & $4.4763 \pm 0.0063$ & $4.4724 \pm 0.0031$ & 4.4709 & 4.4939 \\
103 & 55 & $4.5140 \pm 0.0056$ & $4.4904 \pm 0.0057$ & $4.4869 \pm 0.0031$ & & \\
104 & 56 & $4.5285 \pm 0.0062$ & $4.5071 \pm 0.0053$ & $4.5040 \pm 0.0039$ & 4.5091 & 4.5162 \\
105 & 57 & $4.5364 \pm 0.0048$ & $4.5174 \pm 0.0046$ & $4.5147 \pm 0.0030$ & & \\
106 & 58 & $4.5517 \pm 0.0010$ & $4.5337 \pm 0.0050$ & $4.5324 \pm 0.0026$ & 4.5308 & 4.5362 \\
107 & 59 & $4.5575 \pm 0.0042$ & $4.5428 \pm 0.0037$ & $4.5409 \pm 0.0029$ & & \\
108 & 60 & $4.5673 \pm 0.0010$ & $4.5541 \pm 0.0039$ & $4.5531 \pm 0.0022$ & 4.5457 & 4.5545 \\
109 & 61 & $4.5689 \pm 0.0054$ & $4.5585 \pm 0.0030$ & $4.5575 \pm 0.0044$ & & \\
110 & 62 & $4.5822 \pm 0.0010$ & $4.5736 \pm 0.0029$ & $4.5730 \pm 0.0018$ & 4.5587 & 4.5735 \\
111 & 63 & $4.5845 \pm 0.0010$ & $4.5776 \pm 0.0103$ & $4.5778 \pm 0.0016$ & & \\
$111 \mathrm{~m}$ & 63 & $4.5832 \pm 0.0119$ & $4.5784 \pm 0.0025$ & $4.5767 \pm 0.0111$ & & \\
112 & 64 & $4.5969 \pm 0.0034$ & $4.5926 \pm 0.0019$ & $4.5924 \pm 0.0029$ & 4.5777 & 4.5929 \\
113 & 65 & $4.5993 \pm 0.0010$ & $4.5954 \pm 0.0076$ & $4.5972 \pm 0.0012$ & & \\
$113 \mathrm{~m}$ & 65 & $4.5991 \pm 0.0084$ & $4.5975 \pm 0.0016$ & $4.5950 \pm 0.0083$ & & \\
114 & 66 & $4.6100 \pm 0.0010$ & $4.6100 \pm 0.0010$ & $4.6100 \pm 0.0010$ & 4.5991 & 4.6066 \\
115 & 67 & $4.6125 \pm 0.0059$ & $4.6148 \pm 0.0058$ & $4.6149 \pm 0.0060$ & & \\
$115 \mathrm{~m}$ & 67 & $4.6133 \pm 0.0023$ & $4.6148 \pm 0.0025$ & $4.6156 \pm 0.0024$ & & \\
116 & 68 & $4.6202 \pm 0.0010$ & $4.6245 \pm 0.0018$ & $4.6246 \pm 0.0014$ & 4.6141 & 4.6184 \\
118 & 70 & $4.6235 \pm 0.0034$ & $4.6320 \pm 0.0044$ & $4.6325 \pm 0.0040$ & 4.623 & 4.6305 \\
120 & 72 & $4.6283 \pm 0.0055$ & $4.6408 \pm 0.0070$ & $4.6416 \pm 0.0064$ & 4.6182 & 4.6397 \\
122 & 74 & & & & 4.6301 & 4.6489 \\
124 & 76 & & & & 4.6438 & 4.6595 \\
126 & 78 & & & & 4.6548 & 4.6675 \\
128 & 80 & & & & 4.6652 & 4.6766 \\
130 & 82 & & & & 4.6751 & 4.6945 \\
132 & 84 & & & & 4.702 & 4.7178 \\
134 & 86 & & & & 4.7199 & 4.7372 \\
136 & 88 & & & & & \\
& & & & & \\
\end{tabular}

a) $\delta<\mathrm{r}_{c}^{2}>$ values from ref. [6] and $\mathrm{r}_{c}\left({ }^{114} \mathrm{Cd}\right)$ from ref. [1].

b) $\mathrm{F}=-4.37 \pm 0.18 \mathrm{GHz} / \mathrm{fm}^{2}, \frac{\mathrm{S}}{\mathrm{N}}=2.43 \pm 0.35, \mathrm{k}=0.977$ (see text), IS taken from ref. [12] and $\mathrm{r}_{c}\left({ }^{114} \mathrm{Cd}\right)$ from ref. [1].

c) F from Dirac-Fock calculations $[10,12], \frac{\mathrm{S}}{\mathrm{N}}=2.15 \pm 0.18, \mathrm{k}=0.977$ (see text), IS taken from ref. [12] and $\mathrm{r}_{c}\left({ }^{114} \mathrm{Cd}\right)$ from ref. [1]. 
Table 2

Charge radii (in $\mathrm{fm}$ ) for $\mathrm{Sn}$ isotopes

\begin{tabular}{|c|c|c|c|c|c|c|c|}
\hline $\mathrm{A}$ & $\mathrm{N}$ & $\mathrm{F}_{S E}{ }^{a)}$ & $\mathrm{F}_{\mu 1}{ }^{b)}$ & $\mathrm{F}_{\mu 2}{ }^{c)}$ & $\mathrm{F}_{D F^{d}}{ }^{d)}$ & $\mathrm{HFB}$ & GCM-GOA \\
\hline 100 & 50 & & & & & 4.469 & 4.5033 \\
\hline 102 & 52 & & & & & 4.4823 & 4.5034 \\
\hline 104 & 54 & & & & & 4.497 & 4.5248 \\
\hline 106 & 56 & & & & & 4.5147 & 4.5467 \\
\hline 108 & 58 & & & & & 4.5337 & 4.5653 \\
\hline 110 & 60 & $4.5860 \pm 0.0028$ & $4.5801 \pm 0.0083$ & $4.5748 \pm 0.0115$ & $4.5833 \pm 0.0088$ & 4.5574 & 4.5828 \\
\hline 111 & 61 & $4.5916 \pm 0.0027$ & $4.5860 \pm 0.0074$ & $4.5804 \pm 0.0102$ & $4.5884 \pm 0.0082$ & & \\
\hline 112 & 62 & $4.6013 \pm 0.0022$ & $4.5961 \pm 0.0064$ & $4.5928 \pm 0.0087$ & $4.5993 \pm 0.0070$ & 4.5763 & 4.6001 \\
\hline 113 & 63 & $4.6077 \pm 0.0021$ & $4.6028 \pm 0.0059$ & $4.5999 \pm 0.0081$ & $4.6057 \pm 0.0062$ & & \\
\hline 114 & 64 & $4.6154 \pm 0.0018$ & $4.6108 \pm 0.0048$ & $4.6091 \pm 0.0065$ & $4.6139 \pm 0.0053$ & 4.5928 & 4.6171 \\
\hline 115 & 65 & $4.6203 \pm 0.0017$ & $4.6160 \pm 0.0042$ & $4.6138 \pm 0.0057$ & $4.6183 \pm 0.0048$ & & \\
\hline 116 & 66 & $4.6295 \pm 0.0014$ & $4.6256 \pm 0.0032$ & $4.6257 \pm 0.0042$ & $4.6287 \pm 0.0037$ & 4.6084 & 4.6301 \\
\hline 117 & 67 & $4.6347 \pm 0.0012$ & $4.6310 \pm 0.0026$ & $4.6309 \pm 0.0034$ & $4.6335 \pm 0.0031$ & & \\
\hline $117 \mathrm{~m}$ & 67 & $4.6343 \pm 0.0013$ & $4.6306 \pm 0.0028$ & $4.6303 \pm 0.0038$ & $4.6330 \pm 0.0031$ & & \\
\hline 118 & 68 & $4.6429 \pm 0.0010$ & $4.6396 \pm 0.0017$ & $4.6413 \pm 0.0021$ & $4.6427 \pm 0.0021$ & 4.6229 & 4.6451 \\
\hline 119 & 69 & $4.6475 \pm 0.0008$ & $4.6444 \pm 0.0012$ & $4.6458 \pm 0.0015$ & $4.6468 \pm 0.0017$ & & \\
\hline 120 & 70 & $4.6550 \pm 0.0006$ & $4.6522 \pm 0.0006$ & $4.6550 \pm 0.0006$ & $4.6550 \pm 0.0006$ & 4.6362 & 4.6573 \\
\hline 121 & 71 & $4.6595 \pm 0.0008$ & $4.6570 \pm 0.0013$ & $4.6594 \pm 0.0015$ & $4.6591 \pm 0.0011$ & & \\
\hline $121 \mathrm{~m}$ & 71 & $4.6589 \pm 0.0008$ & $4.6563 \pm 0.0012$ & $4.6584 \pm 0.0014$ & $4.6583 \pm 0.0010$ & & \\
\hline 122 & 72 & $4.6658 \pm 0.0010$ & $4.6635 \pm 0.0023$ & $4.6668 \pm 0.0031$ & $4.6657 \pm 0.0020$ & 4.651 & 4.6693 \\
\hline 123 & 73 & $4.6694 \pm 0.0012$ & $4.6673 \pm 0.0025$ & $4.6696 \pm 0.0032$ & $4.6685 \pm 0.0021$ & & \\
\hline 124 & 74 & $4.6755 \pm 0.0013$ & $4.6737 \pm 0.0035$ & $4.6770 \pm 0.0047$ & $4.6751 \pm 0.0030$ & 4.6622 & 4.6805 \\
\hline 125 & 75 & $4.6791 \pm 0.0016$ & $4.6774 \pm 0.0038$ & $4.6798 \pm 0.0052$ & $4.6779 \pm 0.0032$ & & \\
\hline $125 \mathrm{~m}$ & 75 & $4.6775 \pm 0.0040$ & $4.6758 \pm 0.0037$ & $4.6773 \pm 0.0049$ & $4.6757 \pm 0.0030$ & & \\
\hline 126 & 76 & $4.6846 \pm 0.0051$ & $4.6832 \pm 0.0045$ & $4.6862 \pm 0.0060$ & $4.6836 \pm 0.0039$ & 4.6736 & 4.6911 \\
\hline 127 & 77 & $4.6878 \pm 0.0056$ & $4.6866 \pm 0.0050$ & $4.6888 \pm 0.0067$ & $4.6862 \pm 0.0041$ & & \\
\hline $127 \mathrm{~m}$ & 77 & $4.6858 \pm 0.0053$ & $4.6845 \pm 0.0049$ & $4.6854 \pm 0.0065$ & $4.6833 \pm 0.0038$ & & \\
\hline 128 & 78 & $4.6930 \pm 0.0063$ & $4.6920 \pm 0.0056$ & $4.6946 \pm 0.0076$ & $4.6914 \pm 0.0047$ & 4.6844 & 4.6992 \\
\hline 129 & 79 & $4.6941 \pm 0.0065$ & $4.6933 \pm 0.0060$ & $4.6938 \pm 0.0080$ & $4.6911 \pm 0.0047$ & & \\
\hline $129 \mathrm{~m}$ & 79 & $4.6969 \pm 0.0069$ & $4.6962 \pm 0.0061$ & $4.6984 \pm 0.0081$ & $4.6950 \pm 0.0051$ & & \\
\hline 130 & 80 & $4.7023 \pm 0.0077$ & $4.7018 \pm 0.0067$ & $4.7047 \pm 0.0090$ & $4.7006 \pm 0.0057$ & 4.6946 & 4.7072 \\
\hline $130 \mathrm{~m}$ & 80 & $4.6991 \pm 0.0073$ & $4.6985 \pm 0.0065$ & $4.6994 \pm 0.0086$ & $4.6962 \pm 0.0052$ & & \\
\hline 131 & 81 & $4.7080 \pm 0.0086$ & $4.7077 \pm 0.0073$ & $4.7115 \pm 0.0100$ & $4.7067 \pm 0.0064$ & & \\
\hline $131 \mathrm{~m}$ & 81 & $4.7108 \pm 0.0090$ & $4.7105 \pm 0.0074$ & $4.7161 \pm 0.0102$ & $4.7105 \pm 0.0068$ & & \\
\hline 132 & 82 & $4.7093 \pm 0.0088$ & $4.7091 \pm 0.0075$ & $4.7111 \pm 0.0101$ & $4.7067 \pm 0.0064$ & 4.7043 & 4.7318 \\
\hline 134 & 84 & & & & & 4.7179 & 4.7285 \\
\hline 136 & 86 & & & & & 4.7311 & 4.7476 \\
\hline
\end{tabular}

a) F, SMS and $\mathrm{k}$ from ref. [13], IS from refs. [13,5] and $\mathrm{r}_{c}\left({ }^{120} \mathrm{Sn}\right)$ from ref. [1].

b) F, $\frac{\mathrm{S}}{\mathrm{N}}$ and $\mathrm{k}$ from ref. [5], IS from refs. [13,5] and $\mathrm{r}_{c}\left({ }^{120} \mathrm{Sn}\right)$ from ref. [15].

c) $\mathrm{F}=2.04 \pm 0.2 \mathrm{GHz} / \mathrm{fm}^{2}, \frac{\mathrm{S}}{\mathrm{N}}=-0.78 \pm 0.32$ and $\mathrm{k}=0.975$ (see text), IS from refs. [13,5] and $\mathrm{r}_{c}\left({ }^{120} \mathrm{Sn}\right)$ from ref. [1].

d) F from Dirac-Fock calculations, $\frac{\mathrm{S}}{\mathrm{N}}$ and $\mathrm{k}$ from ref. [16], IS from refs. $[13,5]$ and $\mathrm{r}_{c}\left({ }^{120} \mathrm{Sn}\right)$ from ref. [1]. 
Table 3

Charge radii (in $\mathrm{fm}$ ) for Te isotopes

\begin{tabular}{|c|c|c|c|c|c|}
\hline $\mathrm{A}$ & $\mathrm{N}$ & $\mathrm{F}_{\mu 1}{ }^{a)}$ & $\mathrm{F}_{\mu 2^{b)}}$ & HFB & GCM-GOA \\
\hline 102 & 50 & & & 4.5293 & 4.5476 \\
104 & 52 & & & 4.5716 & 4.5634 \\
106 & 54 & & & 4.576 & 4.5818 \\
108 & 56 & & & 4.597 & 4.6014 \\
112 & 60 & & & 4.6358 & 4.6445 \\
114 & 62 & & & 4.6838 & 4.6709 \\
116 & 64 & & & 4.7234 & 4.6908 \\
118 & 66 & & 4.6777 & 4.701 \\
120 & 68 & $4.7046 \pm 0.0076$ & $4.7020 \pm 0.0073$ & 4.6865 & 4.7048 \\
122 & 70 & $4.7112 \pm 0.0060$ & $4.7098 \pm 0.0056$ & 4.6985 & 4.7111 \\
123 & 71 & $4.7127 \pm 0.0056$ & $4.7114 \pm 0.0051$ & & \\
124 & 72 & $4.7183 \pm 0.0045$ & $4.7180 \pm 0.0042$ & 4.7082 & 4.7182 \\
125 & 73 & $4.7201 \pm 0.0041$ & $4.7200 \pm 0.0036$ & & \\
126 & 74 & $4.7250 \pm 0.0031$ & $4.7258 \pm 0.0028$ & 4.7109 & 4.7272 \\
128 & 76 & $4.7319 \pm 0.0018$ & $4.7339 \pm 0.0016$ & 4.722 & 4.7371 \\
130 & 78 & $4.7388 \pm 0.0005$ & $4.7420 \pm 0.0005$ & 4.7326 & 4.7455 \\
132 & 80 & $4.7456 \pm 0.0029$ & $4.7500 \pm 0.0033$ & 4.7428 & 4.7539 \\
134 & 82 & $4.7517 \pm 0.0041$ & $4.7571 \pm 0.0045$ & 4.7525 & 4.7659 \\
136 & 84 & $4.7729 \pm 0.0077$ & $4.7828 \pm 0.0075$ & 4.7897 & 4.7803 \\
\hline
\end{tabular}

a) $\mathrm{F}=4.66 \pm 0.86 \mathrm{GHz} / \mathrm{fm}^{2}, \frac{\mathrm{S}}{\mathrm{N}}=-1.89 \pm 0.33$ and $\mathrm{k}=0.974$ (see text), IS from refs. $[17,18]$ and $\mathrm{r}_{c}\left({ }^{130} \mathrm{Te}\right)$ from ref. [19].

b) $\mathrm{F}=3.78 \pm 0.48 \mathrm{GHz} / \mathrm{fm}^{2}, \frac{\mathrm{S}}{\mathrm{N}}=-1.73 \pm 0.37$ and $\mathrm{k}=0.974$ (see text), IS from refs. $[17,18]$ and $\mathrm{r}_{c}\left({ }^{130} \mathrm{Te}\right)$ from ref. [1]. 
Table 4

Charge radii (in $\mathrm{fm}$ ) for Xe isotopes

\begin{tabular}{|c|c|c|c|c|c|}
\hline $\mathrm{A}$ & $\mathrm{N}$ & $\mathrm{F}_{S E}{ }^{a)}$ & $\mathrm{F}_{\mu}^{b)}$ & HFB & GCM-GOA \\
\hline 114 & 60 & & & 4.7697 & 4.7136 \\
116 & 62 & $4.7362 \pm 0.0149$ & $4.7075 \pm 0.0073$ & 4.7759 & 4.7408 \\
118 & 64 & $4.7508 \pm 0.0127$ & $4.7291 \pm 0.0057$ & 4.7622 & 4.7571 \\
120 & 66 & $4.7610 \pm 0.0117$ & $4.7439 \pm 0.0046$ & 4.775 & 4.7698 \\
122 & 68 & $4.7677 \pm 0.0095$ & $4.7537 \pm 0.0039$ & 4.8016 & 4.7707 \\
124 & 70 & $4.7737 \pm 0.0085$ & $4.7624 \pm 0.0032$ & 4.8187 & 4.7733 \\
126 & 72 & $4.7788 \pm 0.0064$ & $4.7699 \pm 0.0023$ & 4.7724 & 4.7756 \\
128 & 74 & $4.7831 \pm 0.0053$ & $4.7760 \pm 0.0019$ & 4.7729 & 4.7799 \\
129 & 75 & $4.7831 \pm 0.0043$ & $4.7760 \pm 0.0019$ & & \\
130 & 76 & $4.7868 \pm 0.0043$ & $4.7813 \pm 0.0015$ & 4.7798 & 4.7867 \\
131 & 77 & $4.7861 \pm 0.0032$ & $4.7802 \pm 0.0015$ & & \\
132 & 78 & $4.7902 \pm 0.0022$ & $4.7863 \pm 0.0011$ & 4.7812 & 4.7926 \\
134 & 80 & $4.7936 \pm 0.0014$ & $4.7911 \pm 0.0007$ & 4.7869 & 4.7981 \\
136 & 82 & $4.7990 \pm 0.0001$ & $4.7990 \pm 0.0001$ & 4.7961 & 4.8117 \\
137 & 83 & $4.8099 \pm 0.0011$ & $4.8149 \pm 0.0014$ & & \\
138 & 84 & $4.8254 \pm 0.0022$ & $4.8373 \pm 0.0029$ & 4.8247 & 4.8221 \\
139 & 85 & $4.8363 \pm 0.0032$ & $4.8531 \pm 0.0041$ & & \\
140 & 86 & $4.8494 \pm 0.0042$ & $4.8721 \pm 0.0053$ & 4.8561 & 4.8464 \\
141 & 87 & $4.8602 \pm 0.0052$ & $4.8878 \pm 0.0065$ & & \\
142 & 88 & $4.8724 \pm 0.0063$ & $4.9055 \pm 0.0079$ & 4.8637 & 4.869 \\
143 & 89 & $4.8810 \pm 0.0073$ & $4.9178 \pm 0.0085$ & & \\
144 & 90 & $4.8927 \pm 0.0083$ & $4.9346 \pm 0.0097$ & 4.8814 & 4.889 \\
146 & 92 & $4.9123 \pm 0.0103$ & $4.9628 \pm 0.0117$ & 4.9634 & 4.9141 \\
\hline
\end{tabular}

a) $\delta<\mathrm{r}_{c}^{2}>$ from ref. [20] and $\mathrm{r}_{c}\left({ }^{136} \mathrm{Xe}\right)$ from ref. [1].

b) $\mathrm{F}=-1.56 \pm 0.11 \mathrm{GHz} / \mathrm{fm}^{2}, \frac{\mathrm{S}}{\mathrm{N}}=0.192 \pm 0.266$ and $\mathrm{k}=0.972$ (see text), IS from ref. [20] and $\mathrm{r}_{c}\left({ }^{136} \mathrm{Xe}\right)$ from ref. [1]. 
Table 5

Charge radii (in $\mathrm{fm}$ ) for $\mathrm{Ba}$ isotopes

\begin{tabular}{|c|c|c|c|c|c|c|c|}
\hline $\mathrm{A}$ & $\mathrm{N}$ & $\mathrm{F}_{S E}{ }^{a)}$ & $\mathrm{F}_{\mu 1}{ }^{b)}$ & $\mathrm{F}_{\mu 2}{ }^{c)}$ & $\mathrm{F}_{M C D F^{d)}}$ & $\mathrm{HFB}$ & GCM-GOA \\
\hline 116 & 60 & & & & & 4.8272 & 4.8007 \\
\hline 118 & 62 & & & & & 4.8405 & 4.8249 \\
\hline 120 & 64 & $4.8119 \pm 0.0053$ & & & & 4.8359 & 4.832 \\
\hline 121 & 65 & $4.8200 \pm 0.0051$ & & & & & \\
\hline 122 & 66 & $4.8179 \pm 0.0048$ & $4.8116 \pm 0.0016$ & $4.7966 \pm 0.0032$ & $4.8095 \pm 0.0110$ & 4.8031 & 4.8402 \\
\hline 123 & 67 & $4.8162 \pm 0.0046$ & $4.8090 \pm 0.0016$ & $4.7963 \pm 0.0031$ & $4.8073 \pm 0.0101$ & & \\
\hline 124 & 68 & $4.8209 \pm 0.0043$ & & & & 4.8165 & 4.8417 \\
\hline 125 & 69 & $4.8202 \pm 0.0040$ & $4.8132 \pm 0.0015$ & $4.8033 \pm 0.0027$ & $4.8128 \pm 0.0087$ & & \\
\hline 126 & 70 & $4.8243 \pm 0.0038$ & $4.8180 \pm 0.0014$ & $4.8089 \pm 0.0025$ & $4.8184 \pm 0.0082$ & 4.8361 & 4.8266 \\
\hline 127 & 71 & $4.8226 \pm 0.0035$ & $4.8154 \pm 0.0013$ & $4.8085 \pm 0.0023$ & $4.8163 \pm 0.0073$ & & \\
\hline 128 & 72 & $4.8275 \pm 0.0032$ & $4.8212 \pm 0.0012$ & $4.8149 \pm 0.0021$ & $4.8228 \pm 0.0068$ & 4.8184 & 4.8277 \\
\hline 129 & 73 & $4.8268 \pm 0.0030$ & $4.8200 \pm 0.0011$ & $4.8155 \pm 0.0020$ & $4.8221 \pm 0.0061$ & & \\
\hline $129 \mathrm{~m}$ & 73 & $4.8255 \pm 0.0030$ & $4.8183 \pm 0.0012$ & $4.8141 \pm 0.0021$ & $4.8203 \pm 0.0061$ & & \\
\hline 130 & 74 & $4.8301 \pm 0.0027$ & $4.8237 \pm 0.0010$ & $4.8202 \pm 0.0017$ & $4.8265 \pm 0.0054$ & 4.8194 & 4.8304 \\
\hline 131 & 75 & $4.8294 \pm 0.0025$ & $4.8225 \pm 0.0010$ & $4.8208 \pm 0.0016$ & $4.8257 \pm 0.0047$ & & \\
\hline $131 \mathrm{~m}$ & 75 & $4.8291 \pm 0.0025$ & $4.8220 \pm 0.0010$ & $4.8204 \pm 0.0016$ & $4.8252 \pm 0.0047$ & & \\
\hline 132 & 76 & $4.8320 \pm 0.0022$ & $4.8254 \pm 0.0009$ & $4.8247 \pm 0.0014$ & $4.8292 \pm 0.0041$ & 4.825 & 4.8332 \\
\hline 133 & 77 & $4.8303 \pm 0.0020$ & $4.8228 \pm 0.0009$ & $4.8241 \pm 0.0013$ & $4.8270 \pm 0.0035$ & & \\
\hline $133 \mathrm{~m}$ & 77 & $4.8316 \pm 0.0020$ & $4.8244 \pm 0.0008$ & $4.8254 \pm 0.0013$ & $4.8287 \pm 0.0034$ & & \\
\hline 134 & 78 & $4.8339 \pm 0.0017$ & $4.8265 \pm 0.0007$ & $4.8287 \pm 0.0011$ & $4.8314 \pm 0.0028$ & 4.8265 & 4.8378 \\
\hline 135 & 79 & $4.8313 \pm 0.0016$ & $4.8227 \pm 0.0009$ & $4.8271 \pm 0.0011$ & $4.8279 \pm 0.0022$ & & \\
\hline $135 \mathrm{~m}$ & 79 & $4.8335 \pm 0.0014$ & $4.8261 \pm 0.0007$ & $4.8298 \pm 0.0010$ & $4.8314 \pm 0.0022$ & & \\
\hline 136 & 80 & $4.8350 \pm 0.0011$ & $4.8273 \pm 0.0006$ & $4.8323 \pm 0.0008$ & $4.8332 \pm 0.0016$ & 4.8285 & 4.8403 \\
\hline 137 & 81 & $4.8331 \pm 0.0009$ & $4.8246 \pm 0.0007$ & $4.8316 \pm 0.0008$ & $4.8307 \pm 0.0010$ & & \\
\hline $137 \mathrm{~m}$ & 82 & $4.8394 \pm 0.0004$ & $4.8329 \pm 0.0004$ & $4.8383 \pm 0.0005$ & $4.8395 \pm 0.0009$ & & \\
\hline 138 & 82 & $4.8390 \pm 0.0004$ & $4.8320 \pm 0.0004$ & $4.8390 \pm 0.0004$ & $4.8390 \pm 0.0004$ & 4.8373 & 4.8541 \\
\hline 139 & 83 & $4.8517 \pm 0.0012$ & $4.8481 \pm 0.0012$ & $4.8534 \pm 0.0011$ & $4.8564 \pm 0.0011$ & & \\
\hline 140 & 84 & $4.8676 \pm 0.0022$ & $4.8684 \pm 0.0021$ & $4.8713 \pm 0.0020$ & $4.8782 \pm 0.0017$ & 4.8674 & 4.8634 \\
\hline 141 & 85 & $4.8792 \pm 0.0030$ & $4.8829 \pm 0.0027$ & $4.8845 \pm 0.0026$ & $4.8940 \pm 0.0023$ & & \\
\hline 142 & 86 & $4.8929 \pm 0.0041$ & $4.9002 \pm 0.0035$ & $4.8998 \pm 0.0034$ & $4.9126 \pm 0.0028$ & 4.8792 & 4.8889 \\
\hline 143 & 87 & $4.9055 \pm 0.0049$ & $4.9161 \pm 0.0043$ & $4.9140 \pm 0.0041$ & $4.9297 \pm 0.0035$ & & \\
\hline 144 & 88 & $4.9195 \pm 0.0056$ & $4.9338 \pm 0.0051$ & $4.9297 \pm 0.0048$ & $4.9488 \pm 0.0041$ & 4.9115 & 4.9128 \\
\hline 145 & 89 & $4.9297 \pm 0.0063$ & $4.9466 \pm 0.0056$ & $4.9413 \pm 0.0054$ & $4.9626 \pm 0.0046$ & & \\
\hline 146 & 90 & $4.9424 \pm 0.0071$ & $4.9625 \pm 0.0063$ & $4.9555 \pm 0.0061$ & $4.9797 \pm 0.0051$ & 4.9263 & 4.9414 \\
\hline 148 & 92 & $4.9660 \pm 0.0086$ & & & & 5.0029 & 4.9752 \\
\hline
\end{tabular}

a) $\delta<\mathrm{r}_{c}^{2}>$ from ref. [6] and $\mathrm{r}_{c}\left({ }^{138} \mathrm{Ba}\right)$ from ref. [1].

b) $\mathrm{F}=-3.163 \pm 0.144 \mathrm{GHz} / \mathrm{fm}^{2}, \frac{\mathrm{S}}{\mathrm{N}}=-0.707 \pm 0.109$ and $\mathrm{k}=0.97$ (see text), IS from ref. [22] and $\mathrm{r}_{c}\left({ }^{138} \mathrm{Ba}\right)$ from ref. [21].

c) $\mathrm{F}=-3.897 \pm 0.189 \mathrm{GHz} / \mathrm{fm}^{2}, \frac{\mathrm{S}}{\mathrm{N}}=2.649 \pm 0.27$ and $\mathrm{k}=0.97$ (see text), IS from ref. [22] and $\mathrm{r}_{c}\left({ }^{138} \mathrm{Ba}\right)$ from ref. [1].

d) F from multiconfiguration Dirac-Fock calculations [10], $\frac{\mathrm{S}}{\mathrm{N}}=0.1 \pm 1.1$ and $\mathrm{k}=$ 0.97 (see text), IS from refs. [22] and $\mathrm{r}_{c}\left({ }^{138} \mathrm{Ba}\right)$ from ref. [1]. 
Table 6

Charge radii (in $\mathrm{fm}$ ) for $\mathrm{Sm}$ isotopes

\begin{tabular}{|c|c|c|c|c|c|c|c|}
\hline $\mathrm{A}$ & $\mathrm{N}$ & $\left.\mathrm{F}_{S E^{a}}\right)$ & $\left.\mathrm{F}_{\mu 1}{ }^{b}\right)$ & $\left.\mathrm{F}_{\mu 2}{ }^{c}\right)$ & $\left.\mathrm{F}_{\mu e^{-}}{ }^{d}\right)$ & HFB & GCM-GOA \\
\hline 136 & 74 & & & & & 5.0248 & 4.9716 \\
138 & 76 & $4.9564 \pm 0.0071$ & & & & 4.9633 & 4.9599 \\
139 & 77 & $4.9521 \pm 0.0071$ & & & & \\
140 & 78 & $4.9450 \pm 0.0071$ & & & & & \\
141 & 79 & $4.9447 \pm 0.0078$ & & & & & \\
141 & 79 & $4.9497 \pm 0.0071$ & & & & & \\
142 & 80 & $4.9484 \pm 0.0069$ & & & & & \\
143 & 81 & $4.9447 \pm 0.0071$ & & & & \\
144 & 82 & $4.9490 \pm 0.0060$ & $4.9520 \pm 0.0060$ & $4.9490 \pm 0.0060$ & $4.9490 \pm 0.0060$ & 4.9462 & 4.9637 \\
145 & 83 & $4.9614 \pm 0.0067$ & $4.9636 \pm 0.0076$ & $4.9608 \pm 0.0086$ & $4.9637 \pm 0.0094$ & & \\
146 & 84 & $4.9758 \pm 0.0070$ & $4.9789 \pm 0.0086$ & $4.9768 \pm 0.0106$ & $4.9810 \pm 0.0132$ & 4.9583 & 4.9736 \\
147 & 85 & $4.9857 \pm 0.0088$ & $4.9867 \pm 0.0095$ & $4.9843 \pm 0.0123$ & $4.9927 \pm 0.0158$ & & \\
148 & 86 & $5.0010 \pm 0.0086$ & $5.0035 \pm 0.0106$ & $5.0022 \pm 0.0145$ & $5.0110 \pm 0.0198$ & 4.9893 & 5.0034 \\
149 & 87 & $5.0101 \pm 0.0088$ & $5.0104 \pm 0.0115$ & $5.0086 \pm 0.0162$ & $5.0218 \pm 0.0222$ & & \\
150 & 88 & $5.0312 \pm 0.0090$ & $5.0375 \pm 0.0129$ & $5.0382 \pm 0.0190$ & $5.0473 \pm 0.0278$ & 5.0381 & 5.0373 \\
151 & 89 & $5.0471 \pm 0.0098$ & $5.0560 \pm 0.0141$ & $5.0580 \pm 0.0213$ & $5.0664 \pm 0.0319$ & & \\
152 & 90 & $5.0730 \pm 0.0096$ & $5.0916 \pm 0.0157$ & $5.0971 \pm 0.0247$ & $5.0979 \pm 0.0387$ & 5.1125 & 5.0964 \\
153 & 91 & $5.0825 \pm 0.0060$ & $5.0995 \pm 0.0166$ & $5.1049 \pm 0.0262$ & $5.1091 \pm 0.0412$ & & \\
154 & 92 & $5.0956 \pm 0.0098$ & $5.1137 \pm 0.0176$ & $5.1198 \pm 0.0282$ & $5.1248 \pm 0.0446$ & 5.1215 & 5.1176 \\
\hline
\end{tabular}

a) $\delta<\mathrm{r}_{c}^{2}>$ from ref. [6] and $\mathrm{r}_{c}\left({ }^{144} \mathrm{Sm}\right)$ from ref. [1].

b) $\mathrm{F}=-2.82 \pm 0.14 \mathrm{GHz} / \mathrm{fm}^{2}, \frac{\mathrm{S}}{\mathrm{N}}=-16.37 \pm 1.84$ and $\mathrm{k}=0.963$ (see text), IS from ref. [25] and $\mathrm{r}_{c}\left({ }^{144} \mathrm{Sm}\right)$ from ref. [27].

c) $\mathrm{F}=-2.48 \pm 0.23 \mathrm{GHz} / \mathrm{fm}^{2}, \frac{\mathrm{S}}{\mathrm{N}}=-18.79 \pm 3.01$ and $\mathrm{k}=0.963$ (see text), IS from ref. [25] and $\mathrm{r}_{c}\left({ }^{144} \mathrm{Sm}\right)$ from ref. [1].

d) $\mathrm{F}$ and $\frac{\mathrm{S}}{\mathrm{N}}$ from combined analysis [1] and $\mathrm{r}_{c}\left({ }^{144} \mathrm{Sm}\right)$ from ref. [1].

\section{References}

[1] G. Fricke, C. Bernhardt, K. Heilig, L.A. Schaller, L. Schellenberg, E.B. Shera and C.W. de Jager, Atomic Data and Nuclear Data Tables 60 (1995) 177.

[2] J. Sauvage, J. Libert, B. Roussière, F. Le Blanc, J. Oms, A. Ouchrif, D. Verney, Rapport d'activité IPNO 2000/2001 p. 32.

[3] B. Roussière, VI Latin American Symposium on Nuclear Physics and Applications, SLAFNAP6, October 3-7, 2005, Iguazu, Argentina, to be published in American Institute of Physics.

[4] K. Bennaceur, P. Bonche and J. Meyer, C. R. Physique 4 (2003) 555.

[5] F. Le Blanc et al., Phys. Rev. C 72 (2005) 034305. 
[6] E.W. Otten, in Treatise in Heavy-Ion Physics, vol. 8, 1989, p. 517 and refs quoted in.

[7] H.-J. Kluge and W. Nortershauser, Spectrochimica Acta Part. B 58 (2003) 1031, and refs quoted in.

[8] K. Heilig and H. Steudel, Atomic Data and Nuclear Data Tables 14 (1974) 613.

[9] E.C. Seltzer, Phys. Rev. 188 (1969) 1916.

[10] G. Torbohm, B. Fricke and A. Rosén, Phys. Rev. A 31 (1985) 2038.

[11] W.H. King, Isotope shifts in atomic spectra, Plenum Press, New York and London (1984).

[12] F. Buchinger, P. Dabkiewicz, H.-J. Kluge, A.C. Mueller and E.W. Otten, Nucl. Phys. A 462 (1987) 305.

[13] M. Anselment, K. Bekk, A. Hanser, H. Hoeffgen, G. Meisel, S. Göring, H. Rebel, and G. Schatz, Phys. Rev C 34 (1986) 1052.

[14] P.E.G. Baird et al., J. Phys. B 16 (1983) 2485.

[15] C. Piller, C. Gugler, R. Jacot-Guillarmod, L.A. Schaller, L. Schellenberg, H. Schneuwly, G. Fricke, T. Hennemann and J. Herberz, Phys. Rev. C 42 (1990) 182.

[16] J. Eberz, U. Dinger, G. Huber, H. Lochman, R. Menges, G. Ulm, R. Kirchner, O. Klepper, T.U. Kühl and D. Marx, Z. Phys. A 326 (1987) 121.

[17] R. Sifi et al., VII International Workshop on Application of lasers in atomic nuclei research, LASER2006, May 29-June 01, 2006, Poznan, Poland.

[18] B. Roussière et al., ISOLDE Workshop, February 6-8, 2006, CERN, Geneva, Switzerland.

[19] E.B. Shera, M.V. Hoehn, G. Fricke and G. Mallot, Phys. Rev. C 39 (1989)195.

[20] W. Borchers, E. Arnold, W. Neu, R. Neugart, K. Wendt and G. Ulm, Phys. Let. B 216 (1989) 7.

[21] E.B. Shera, H.B. Wohlfahrt, M.V. Hoehn and Y. Tanaka, Phys. Let. B 112 (1982) 124.

[22] K. Bekk, A. Andl, S. Göring, A. Hanser, G. Nowicki, H. Rebel and G. Schatz, Z. Phys. A 291 (1979) 219.

[23] A.C. Mueller, F. Buchinger, W. Klempt, E.W. Otten, R. Neugart, C. Ekström and J. Heinemeier, Nucl. Phys. A 403 (1983) 234.

[24] V.S. Letokhov, V.I. Mishin, S.K. Sekatsky, V.N. Fedoseyev, G.D. Alkhazov, A.E. Barzakh, V.P. Denisov and V.E. Starodubsky, J. Phys. G 18 (1992) 1177.

[25] J.G. England, I.S. Grant, J.A. Griffith, D.E. Evans, D.A. Eastham, G.W.A. Newton and P.M. Walker, J. Phys. G 16 (1990) 105. 
[26] H. Brand, B. Seibert and A. Steudel, Z. Phys. A 296 (1980) 281.

[27] R.J. Powers, P. Barreau, B. Bihoreau, J. Miller, J. Morgenstern, J. Picard and L. Roussel, Nucl. Phys. A 316 (1979) 295.

[28] P. Barreau, L. Roussel and R.J. Powers, Nucl. Phys. A 364 (1981) 446.

[29] I. Angeli, Atomic Data and Nuclear Data Tables 87 (2004) 185.

[30] D. Gogny, in Proceedings of the International Conference on Nuclear Physics, Munich, 1973, edited by J. De Boer and H.J. Mang (North Holland, Amsterdam, 1973); in Proceedings of the International Conference on Nuclear Selfconsistent Fields, Trieste, 1975, edited by G. Ripka and M. Porneuf (North Holland, Amsterdam, 1975).

[31] J. Dechargé and D. Gogny, Phys. Rev. C 21 (1980) 1568.

[32] J.-F. Berger, M. Girod and D. Gogny, Comput. Phys. Commun. 63 (1991) 365.

[33] M. Girod and B. Grammaticos, Phys. Rev. C 27 (1983) 2317.

[34] M. Bertozzi et al. Phys. Lett. B 41 (1972) 408.

[35] J.-P. Delaroche et al. Phys. Rev. C 50 (1994) 2332.

[36] J. Libert, M. Girod and J.-P. Delaroche Phys. Rev C 60 (1999) 054301.

[37] J.-P. Delaroche, M. Girod, H. Goutte, J. Libert Nucl. Phys. A 771 (2006) 107.

[38] K. Kumar and M. Baranger Nucl. Phys A 92 (1967) 608

[39] S. Raman, C.W. Nestor and P. Tikkanen, Atomic Data and Nuclear Data Tables 78 (2001) 64

[40] http://www.nndc.bnl.gov/ensdf/za_form.jsp 

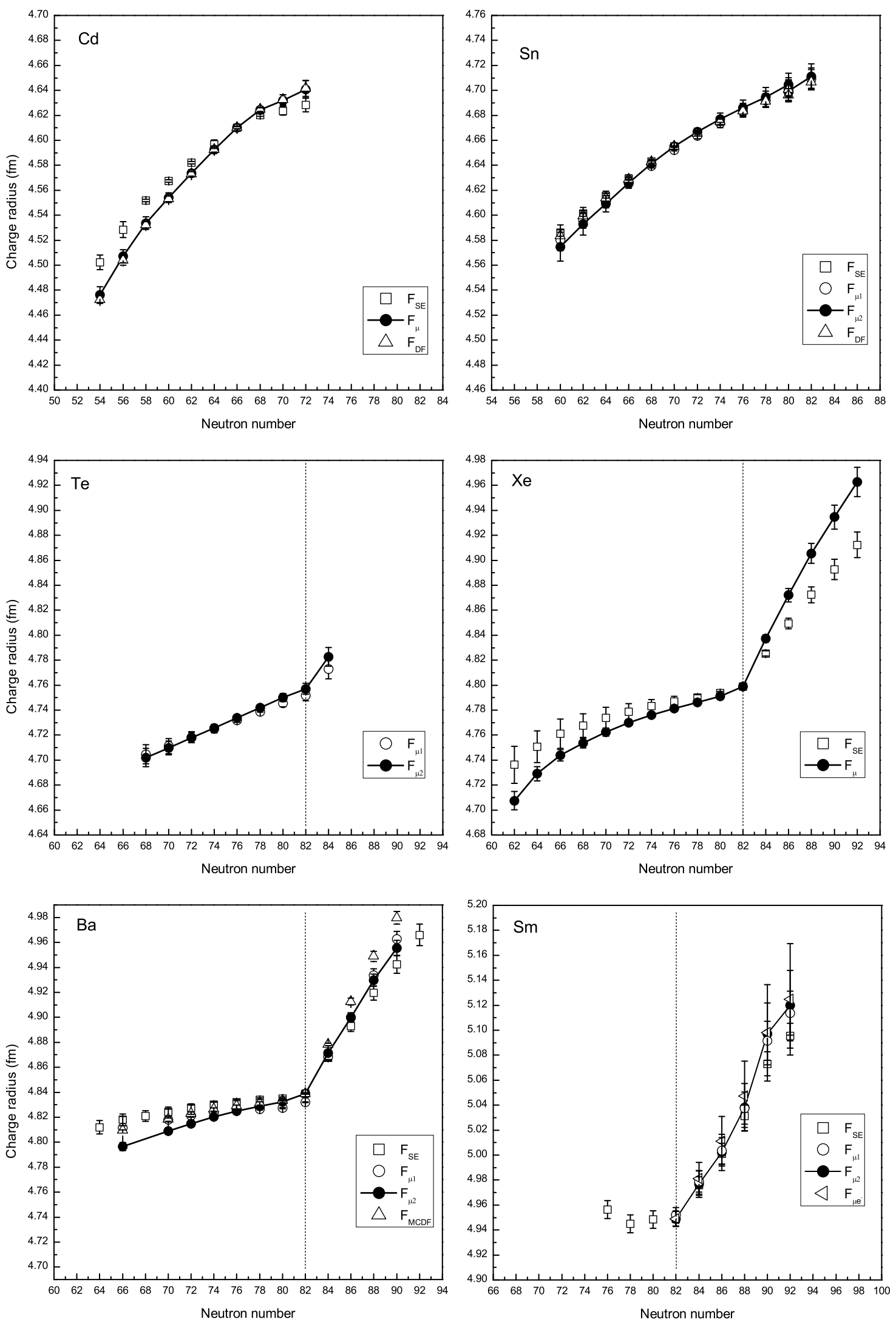

Fig. 1. Experimental charge radius values in the even-even $\mathrm{Cd}, \mathrm{Sn}, \mathrm{Te}, \mathrm{Xe}, \mathrm{Ba}$ and Sm isotopes (see text). 


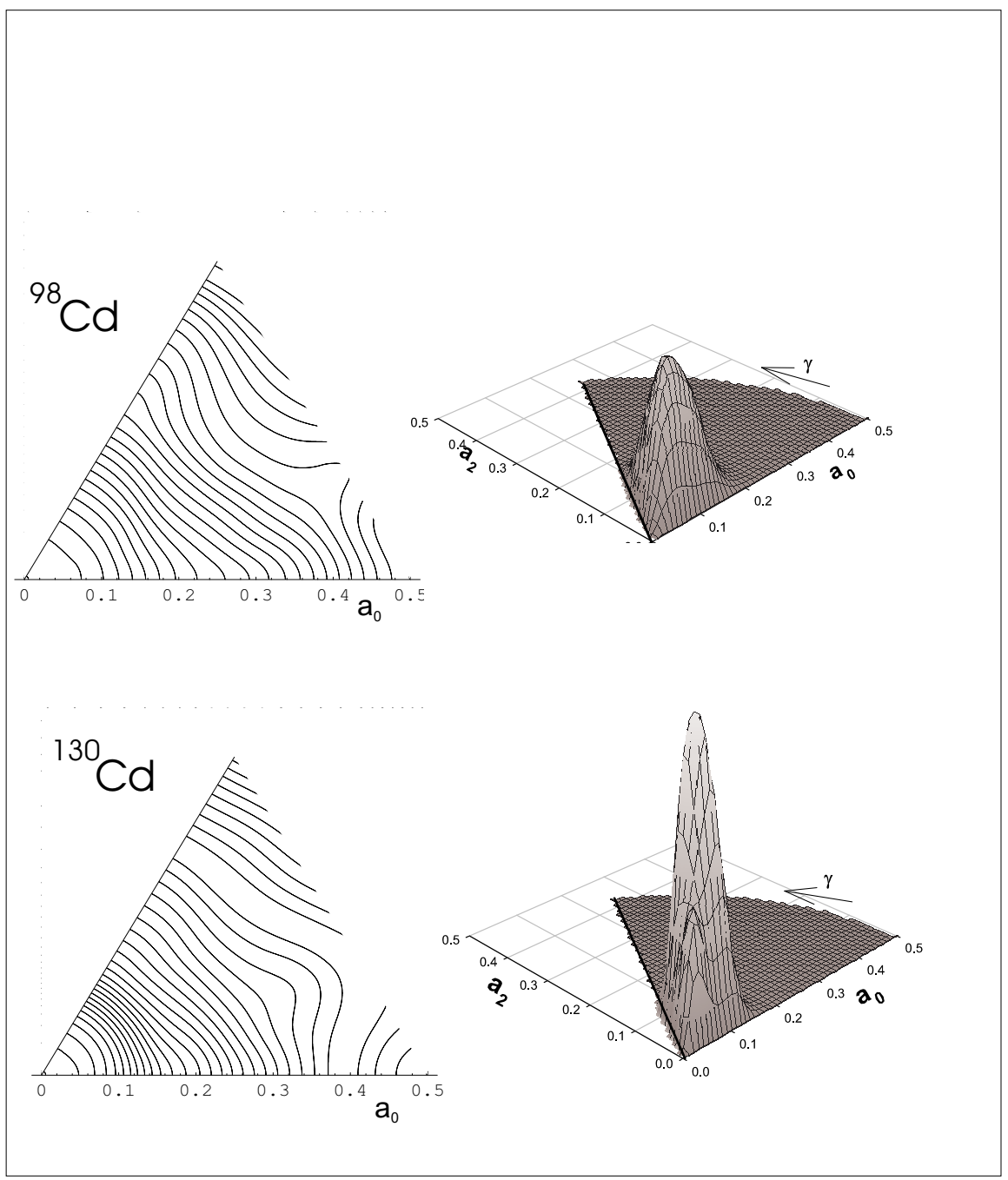

Fig. 2. Potential Energy Surfaces (left hand side) and densities of probability in the ground state (right hand side) -see text- for magic ${ }^{98} \mathrm{Cd}(\mathrm{N}=50)$ and ${ }^{130} \mathrm{Cd}$ $(\mathrm{N}=82)$ nuclei. Equipotential lines are separated by $1 \mathrm{MeV}$. Vertical scales for densities $\rho^{0,1}$ are the same for both nuclei. Maximum value of $\rho^{0,1}$ is 116 (resp. 228) for ${ }^{98} \mathrm{Cd}$ (resp. ${ }^{130} \mathrm{Cd}$ ). 


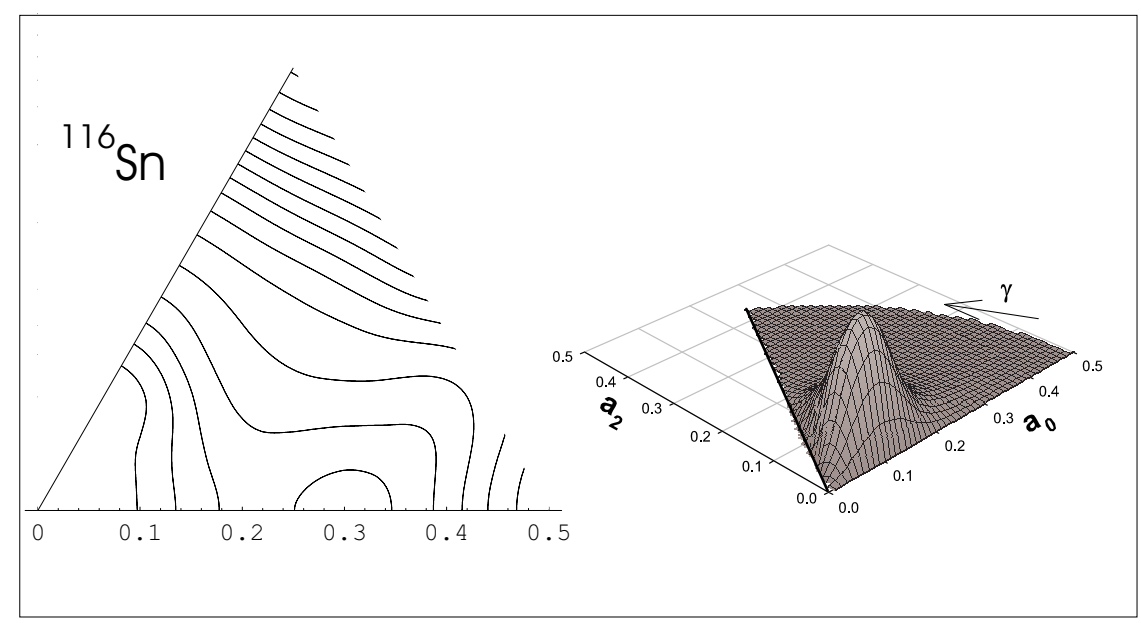

Fig. 3. Same as fig. 2 for the mid shell nucleus ${ }^{116} \mathrm{Sn}(\mathrm{N}=66)$. Vertical scale for potential $\mathrm{V}$ and density $\rho^{0,1}$ are the same as in fig. 2. Maximum value of $\rho^{0,1}$ is 88 for this nucleus. 


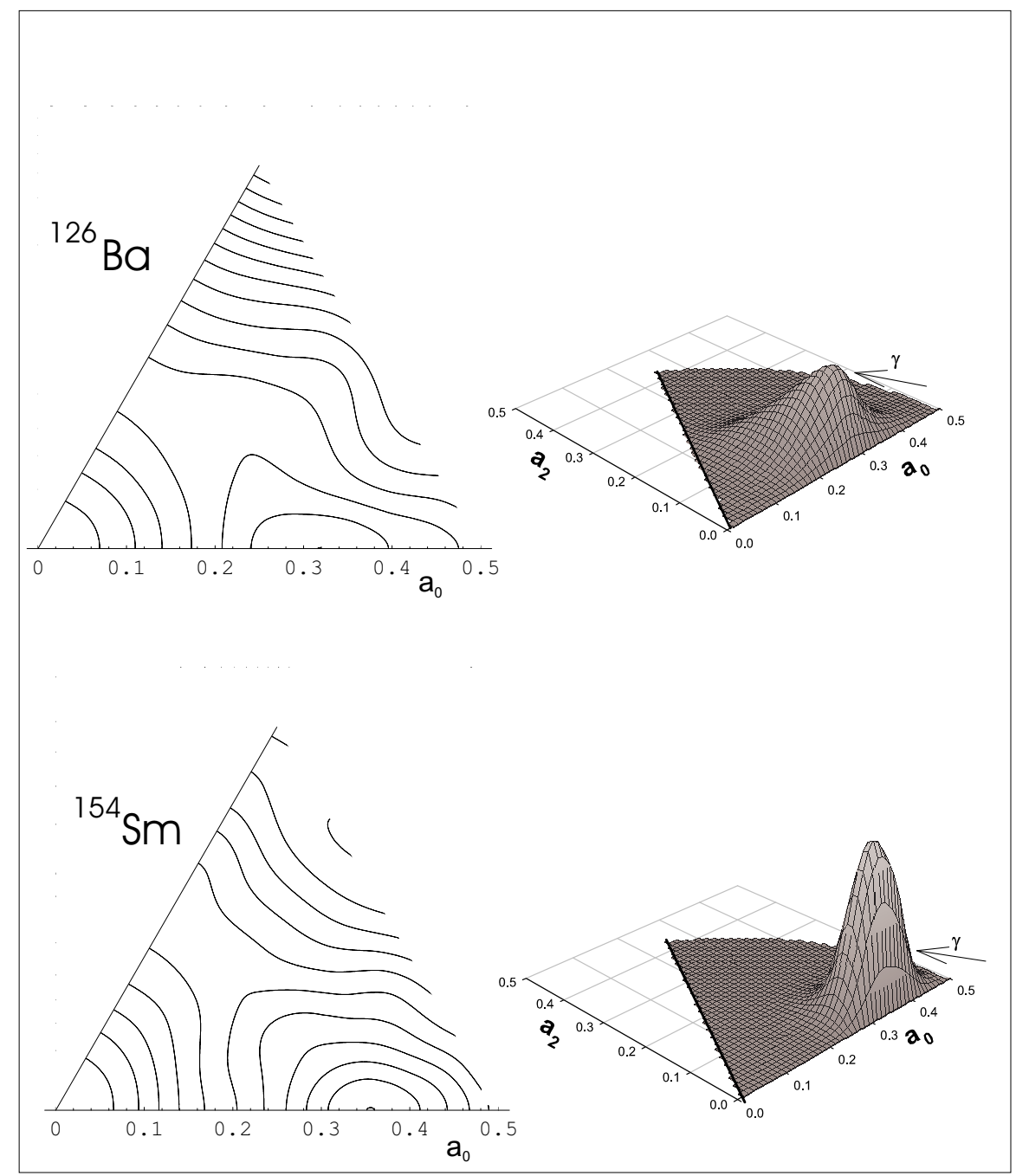

Fig. 4. Same as fig. 2 for the two prolate well deformed nuclei ${ }^{126} \mathrm{Ba}$ and ${ }^{154} \mathrm{Sm}$. Vertical scales for potentials $\mathrm{V}$ and densities $\rho^{0,1}$ are the same as in fig. 2. Maximum value of $\rho^{0,1}$ is 65 (resp. 136) for ${ }^{126} \mathrm{Ba}$ (resp. ${ }^{154} \mathrm{Sm}$ ). 

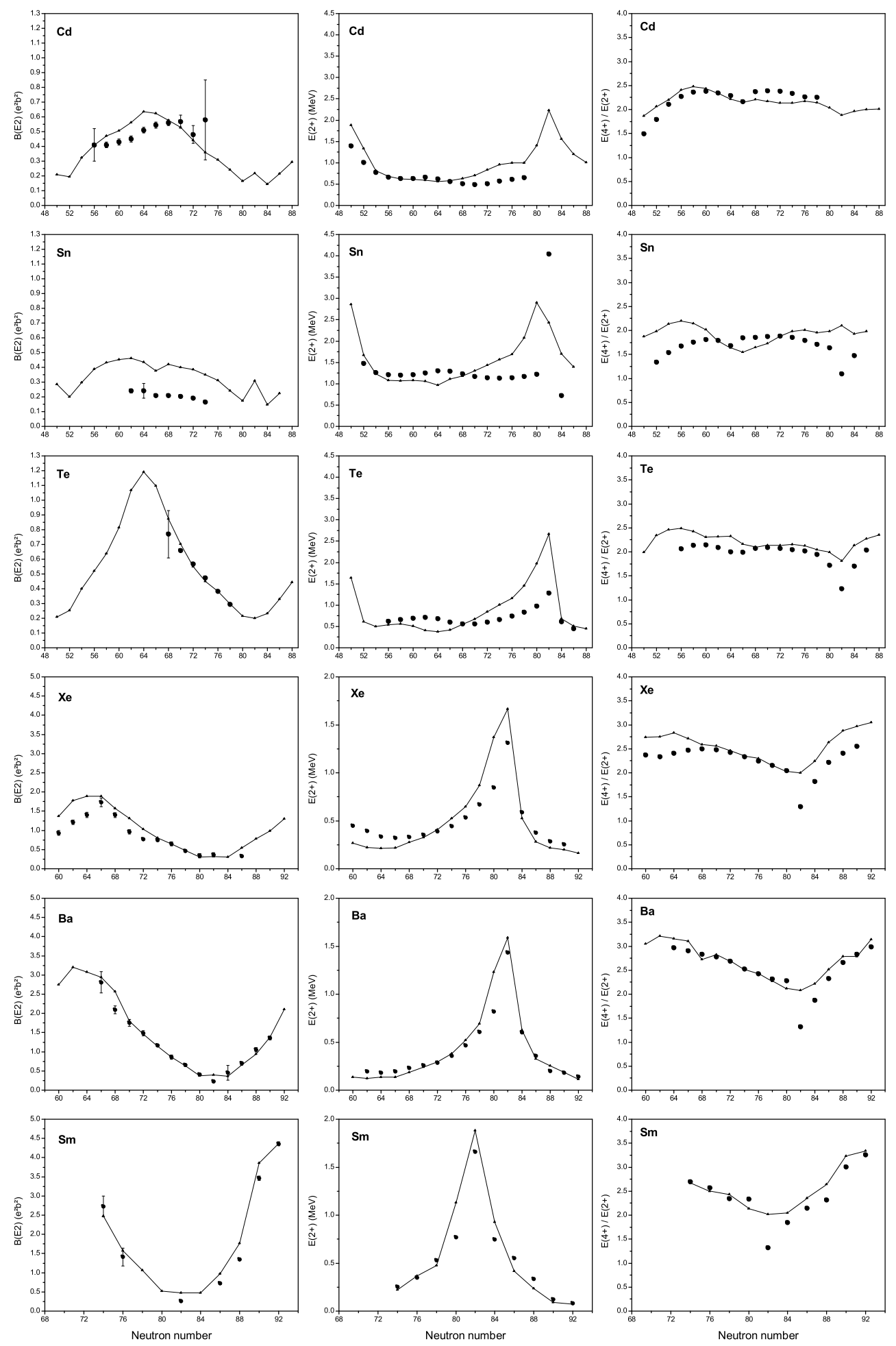

Fig. 5. GCM-GOA calculated quantities (full lines and triangles) are compared with experimental values (full dots with error bars for $\mathrm{B}(\mathrm{E} 2)$ values) in each isotopic chain from successively Cd (upper part), Sn, Te, Xe, Ba to Sm (lower part). This comparison is displayed for transition probabilities $\mathrm{B}\left(\mathrm{E} 2 ; 0_{g s}^{+} \rightarrow 2_{g s}^{+}\right)$(left column), excitation energy $\mathrm{E}\left(2_{g s}^{+}\right)$of the first excited state (central column) and the ratio of excitation energies $\mathrm{E}\left(4_{g s}^{+}\right) / E\left(2_{g s}^{+}\right)$as a measurement of the vibrational-rotational character of the spectrum. The experimental data have been taken from refs. $[39,40]$. 

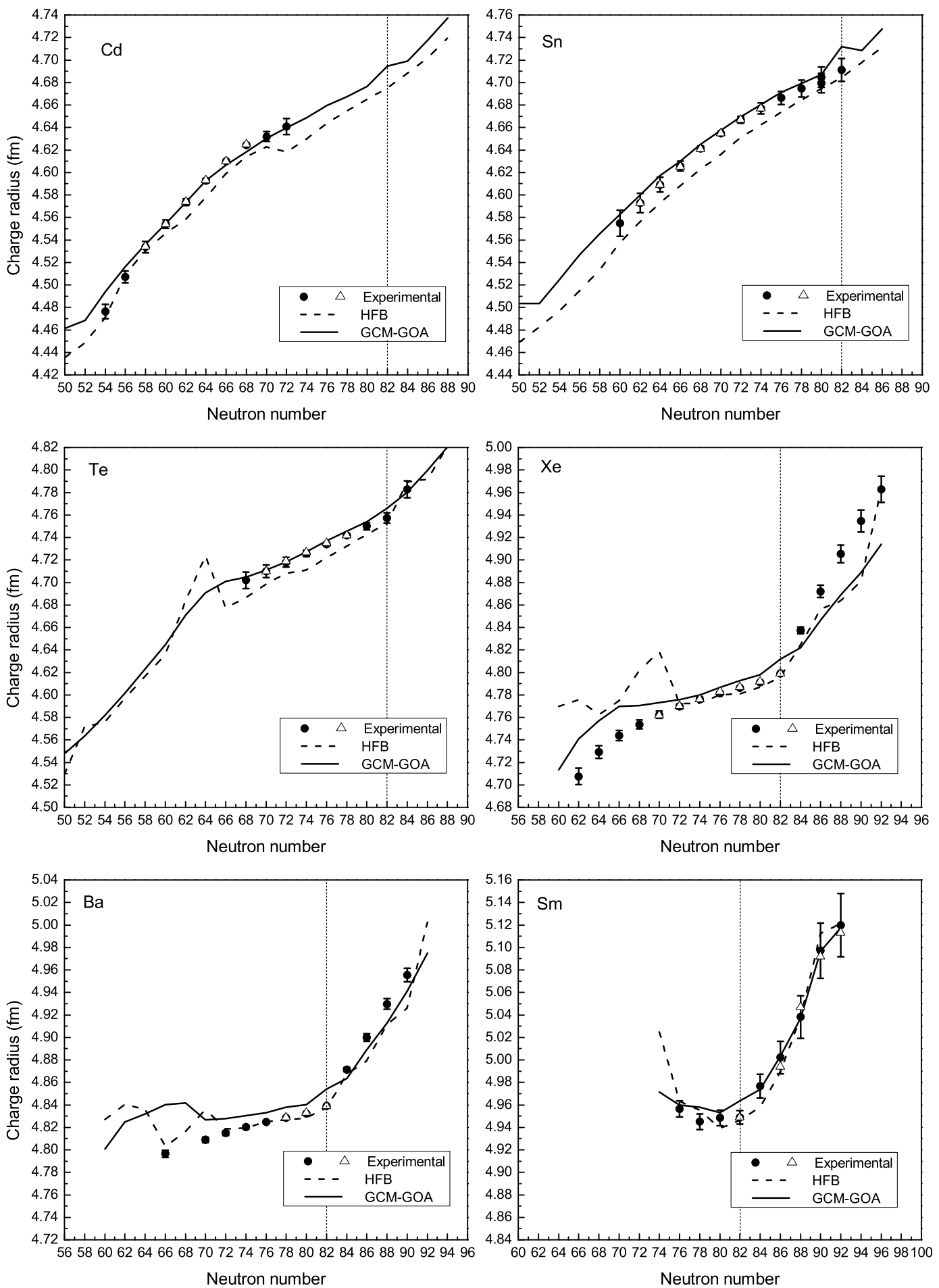

Fig. 6. Experimental and calculated charge radii in even even $\mathrm{Cd}, \mathrm{Sn}, \mathrm{Te}, \mathrm{Xe}, \mathrm{Ba}$ and $\mathrm{Sm}$ isotopes. The experimental $\mathrm{r}_{c}$ values given in ref. [1] for even even nuclei are shown as open triangles. 


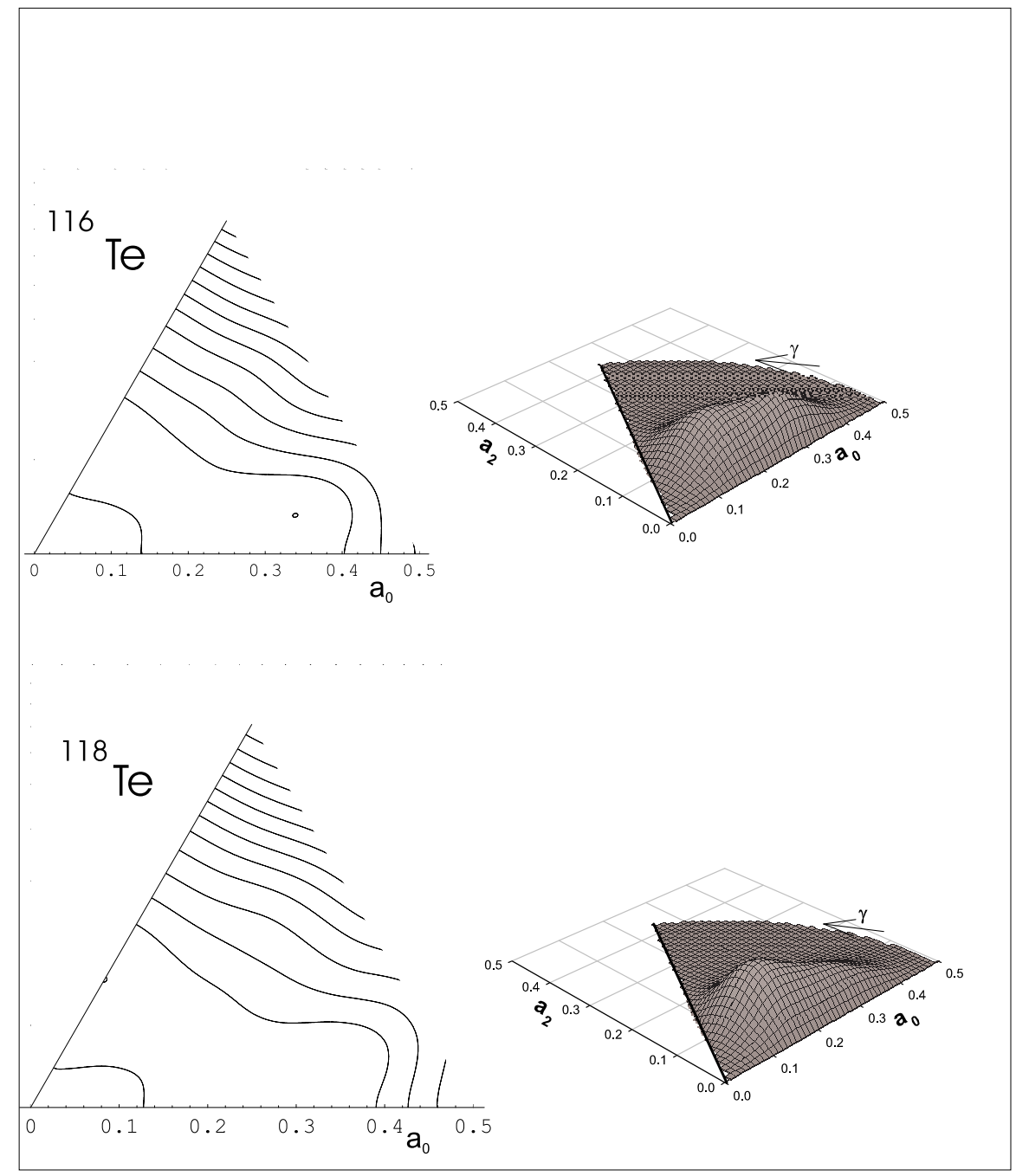

Fig. 7. Same as fig. 2 for ${ }^{116-118}$ Te. Vertical scales for potentials $\mathrm{V}$ and densities $\rho^{0,1}$ are the same as in fig. 2. Maximum value of $\rho^{0,1}$ is 38 (resp. 45) for ${ }^{116} \mathrm{Te}$ (resp. ${ }^{118} \mathrm{Te}$ ). 


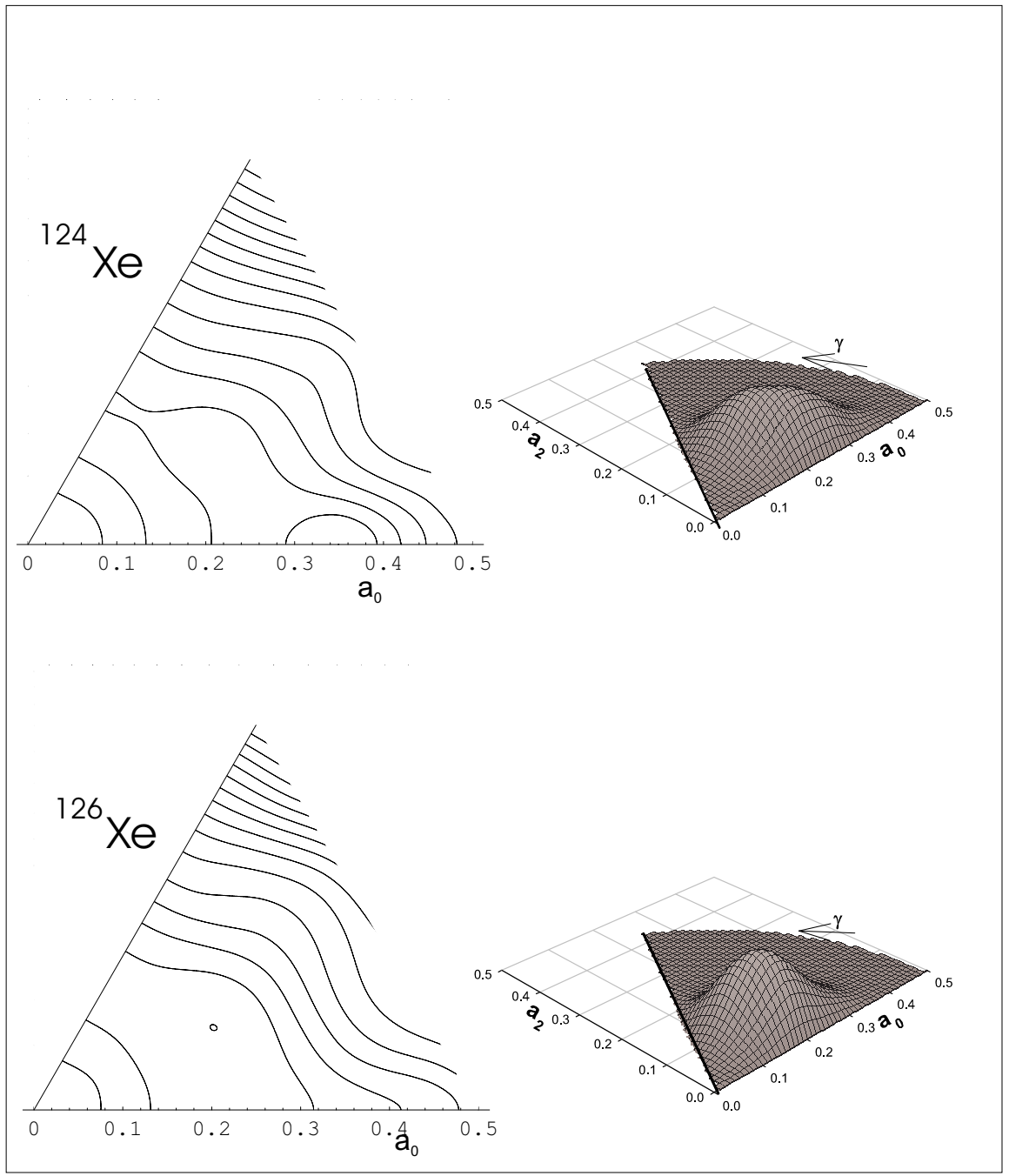

Fig. 8. Same as fig. 2 for ${ }^{124-126}$ Xe.Vertical scales in potentials $\mathrm{V}$ and densities $\rho^{0,1}$ are the same as in fig. 2. Maximum value of $\rho^{0,1}$ is 46 (resp. 59) for ${ }^{124} \mathrm{Xe}$ (resp. $\left.{ }^{126} \mathrm{Xe}\right)$. 

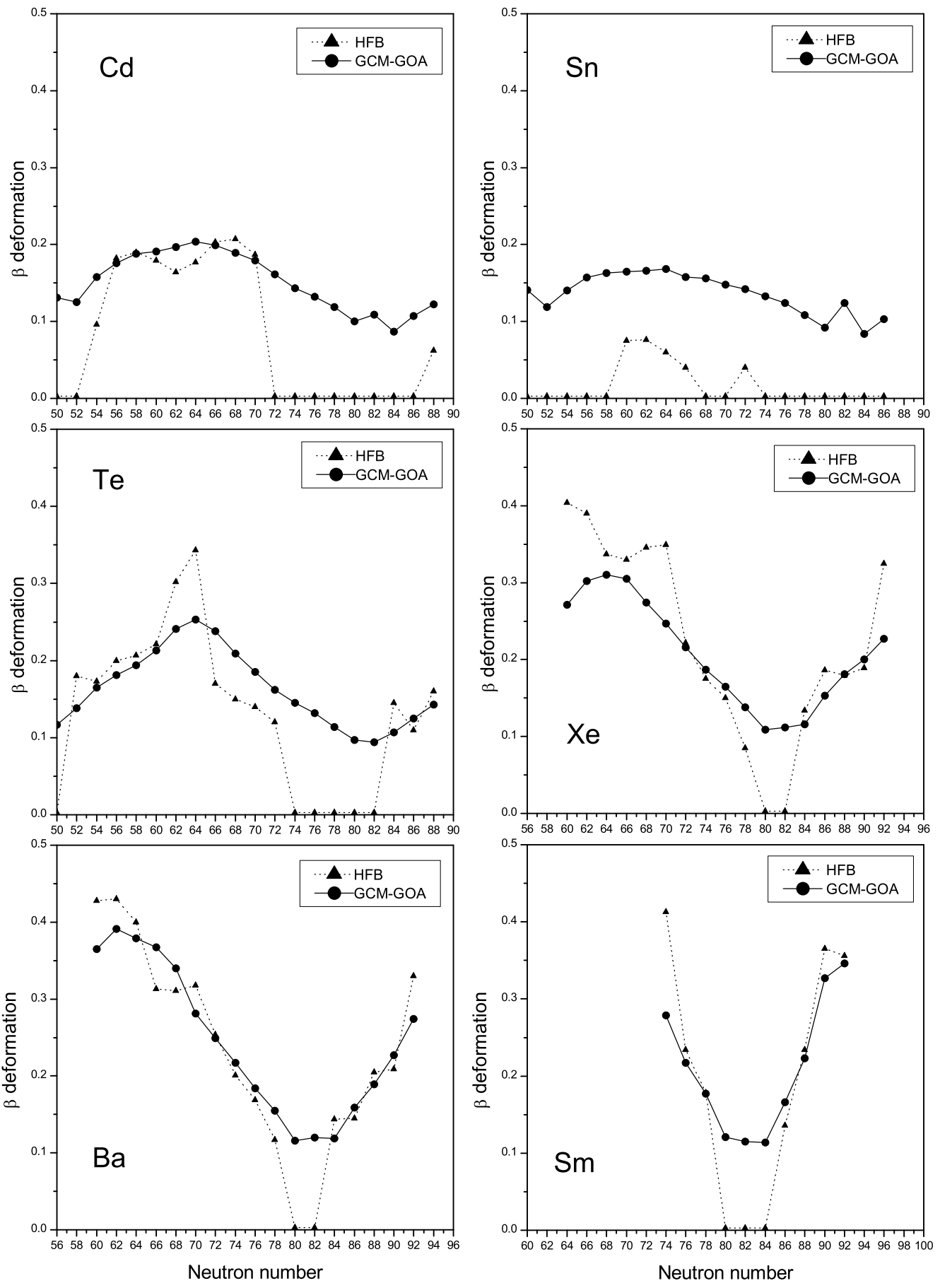

Fig. 9. Theoretical (HFB and GCM-GOA) deformation parameter $\beta$ for the ground state against the neutron number $\mathrm{N}$ in even even $\mathrm{Cd}, \mathrm{Sn}, \mathrm{Te}, \mathrm{Xe}, \mathrm{Ba}$ and $\mathrm{Sm}$ isotopes. 

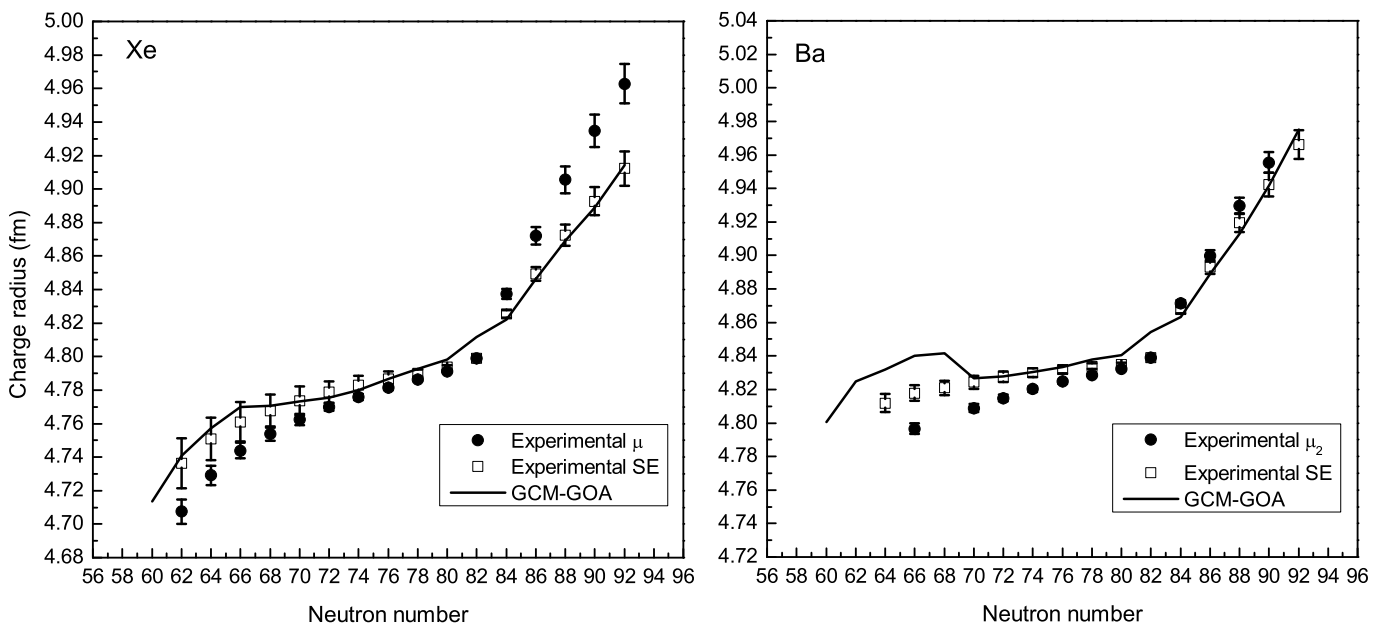

Fig. 10. For Xe (left hand side) and Ba (right hand side) nuclei, charge radii deduced from semi-empirical method (open squares) and with the King plot on the ground of muonic atom results (full dots) are compared with dynamical charge radii provided by the GCM-GOA approach (full line). 